\title{
Joint Topical Word Embedding for Detecting Drift in Social Media Text
}

\author{
Vijayarani $\mathrm{J}^{1}$, Geetha $\mathrm{T} \mathrm{V}^{2}$ \\ 1viji.cs66@gmail.com, ${ }^{2}$ tv_g@ hotmail.com
}

\begin{abstract}
Social media texts like tweets and blogs are collaboratively created by human interaction. Fast change in trends leads to topic drift in the social media text. This drift is usually associated with words and hashtags. However, geotags play an important part in determining topic distribution with location context. Rate of change in the distribution of words, hashtags and geotags cannot be considered as uniform and must be handled accordingly. This paper builds a topic model that associates topic with a mixture of distributions of words, hashtags and geotags. Stochastic gradient Langevin dynamic model with varying mini-batch sizes is used to capture the changes due to the asynchronous distribution of words and tags. Topical word embedding with co-occurrence and location contexts are specified as hashtag context vector and geotag context vector respectively. These two vectors are jointly learned to yield topical word embedding vectors related to tags context. Topical word embeddings over time conditioned on hashtags and geotags predict, location-based topical variations effectively. When evaluated with Chennai and UK geolocated Twitter data, the proposed joint topical word embedding model enhanced by the social tags context, outperforms other methods.
\end{abstract}

Keywords: topic drift, hashtag, geotag, Langevin dynamics, word embedding, topic2vec

\section{INTRODUCTION}

Topics identified in a document make sense to the group of words. Topic drift is different from semantic drift or semantic change from the perspective that the first one is related to the change in the group wise distribution of words over time, where the second one reflects the change in the usage of words over time. Tweets and micro blogs have diverse contents like text, urls, hashtags, geotags, mentions, image, emoticons and time stamp. User groups and user

${ }^{1}$ Corresponding author

${ }^{1,2}$ Department of CSE, CEG, Anna University, Chennai 
interests are connected with the locations and have faster dynamics based on location specific events. Words and hashtags distributions in tweets fluctuate at regular intervals during festivals (such as Diwali and Christmas) and sports events (such as Olympics). These changes may or may not lead to the topic drift. Hence, the topic drift in tweets is a complex issue, and must be processed with a different perspective.

From literature, it is evident that the topic drift can be examined by modeling time with word co-occurrence patterns (Wang \& McCallum 2006), identifying topic boundaries (Liu et al. 2013), detecting the sub topics (Fei et al. 2015), quantifying the impact of the topic on a location (Bernabe-Moreno et al. 2015) and representing the context as a cluster of hashtags (Alam et al. 2017). Social media text reflects a cultural change in the social environment that leads to topic drift where location plays a major role. Geotagged tweets reflect the behavior of people in that region. Location proximity relates messages with the same event in addition to time (Atefeh \& Khreich 2015) and used for detecting location based topical variation. Zhang et al. (2015) combined clustering and topic model to study topic clusters of documents from a geolocation. Lozano et al. (2017) studied how topics correlate with the location and topical locations change over time. Words and hashtags are useful for identifying short term topical variation. But, the geolocation from which the user posted tweets has a greater impact on the topic distribution compared to words and hashtags. Hence, geolocations should also be considered for investigating the long term location based contextual change (Bernabe-Moreno et al. 2015).

The most commonly used methods for modelling topics is latent Dirichlet allocation (LDA), which represents the discrete unstructured text document as random mixtures over latent topics and topic as a distribution of words (Blei et al. 2003). There is a possibility of grouping a single word under two or more topics with different probabilities at different time. There are a variety of modifications of LDA such as, labeled LDA (Ramage et al. 2009), Twitter LDA (Zhao et al. 2011), author topic model (Rosen-Zvi et al. 2004) and hashtag based topic model (Alam et al. 2017; Wang et al. 2014; Wang et al. 2016). However, hashtags used in tweets have faster dynamic nature compared to the locations associated with the tweets. Hence, LDA for Twitter data should incorporate these additional attributes, which are conditionally independent and distributed in an asynchronous manner. 
The evolving nature of the contents of social media text is tremendously high. Hence, a suitable dynamic modeling approach is required for capturing the topic dynamics from a large number of tweets that are partitioned across either discrete or continuous time slots. The dynamic topic models (DTM) described, using the variational Kalman filtering and non-parametric wavelet regression (Blei \& Lafferty 2006), online inference using a stochastic EM algorithm (Iwata et al. 2010), Gibbs sampling with stochastic gradient Langevin dynamics (Bhadury et al. 2016) and continuous time dynamic topic model using Brownian motion (Wang et al. 2006) illustrate how the posterior inference of topics over time can be performed effectively. Continuous time dynamic topic models are suitable for generating topics from sequential collection of documents (Wang et al. 2006) and may not be appropriate for tweets because, Twitter APIs cannot be used to find tweets older than a week. In addition to that, topic in tweets depends on multiple attributes with varying dynamic distributions. Hence, the dynamic topic model used for Twitter data should be discrete (Bhadury et al. 2016) that is capable of integrating varying dynamic behavior of words, tags, urls and mentions over time.

Word embeddings (WEs) extended with topics (Niu et al. 2015; Liu et al. 2015; Li et al. 2016; Qiang et al. 2017; Xun et al. 2016; Jiang et al. 2016) show different ways of integrating topic and word embedding models. Topical word embedding (TWE) model for Twitter data should incorporate additional contexts related to the mixture of distribution of words, tags, urls, mentions and geolocations. Hashtags are the labels for tweets that share a common topic, which are generated by the internet users to categorize the concepts. Hashtags are associated with the co-occurrence context. Geotag is a metadata that describes the geographic location of tweets and is connected with location contexts. These tags enable the achievement of high quality topical word embeddings enhanced with hashtags and geolocations. These embeddings can be learned incrementally over time for investigating the topic drift in tweets.

The proposed model include, a tags context-based topic model to generate the topic distribution over words conditioned on hashtags and geotags and, a discrete time stochastic gradient Langevin dynamic model with varying mini-batch sizes for capturing the changes in the asynchronous distribution of words, hashtags and geotags. Another contribution of this paper is, a topical word embedding model that enhances the proposed topic model to yield topical word embedding vectors trained on contexts related to hashtags and geotags. 


\section{RELATED WORK}

The methods used for analyzing the topic drift, existing topic models for the twitter data, the available discrete time dynamic topic models and the use of topical word embedding are presented.

\subsection{Topic Drift in Twitter Data}

Cataldi et al. (2010) extracted terms from tweets, estimated user authority based on a directed graph of active authors, computed 'term life cycle' using aging theory and selected emerging terms based on its age. They constructed a 'topic graph' that links emerging terms with their co-occurring terms. Emerging term connected with the semantically related words lead to a subgraph of the topic graph showing drift in topics. Saha \& Sindhwani (2012) employed online nonnegative matrix factorization (NMF) to generate topics of streaming text like blogs and tweets, and formulated a temporal regularization operator for topic evolution. Fei et al. (2015) proposed 'cluster based subtopic detection algorithm' to cluster the tweets into subtopics for examining topic drift. However, hashtags are not considered in analyzing the drift. Rosa et al. (2011) performed supervised topical clustering of tweets into predefined categories using hashtags as topic indicators. After clustering at coarse and fine levels, they observed the difference between clusters in training and testing data. Alam et al. (2017) represented topic as 'word distribution' and context as 'hashtags distribution' and studied the context over time with continuous and discrete time distribution. Time attribute for topic evolution can be directly added to the topic model (Wang \& McCallum 2006) for continuous time distribution. Liu et al. (2013) employed LDA with Gibbs sampling for extracting topic words and measured coherence of topical content as change in alignment of topic word's sequence over time. However, the other topic indicators such as urls, geolocations and mentions have not been considered. The location context has impact on topic distribution equivalent to the co-occurrence context with hashtags. Bernabe-Moreno et al. (2015) studied the user interactions with topic and investigated the impact of the topic in a location over a period of time in tweets. However, each location has its own influence on the topic distribution in tweets and the location attribute should be integrated into the topic model for extracting topics from tweets.

\subsection{Extention of Topic Models for Tweets}


Labeled LDA (Ramage et al. 2009, 2010) was described by categorizing the twitter content into four types of dimensions like substance, social, style and status using hashtags, mentions and emoticons in tweets. Zhao et al. (2011) proposed Twitter LDA for generating topics from tweets by distinguishing the word distributions as 'general words' and 'topic words' and categorized topics as longstanding, entity-oriented and event-oriented for opinion mining. Mehrotra et al. (2013) enhanced LDA for tweets by aggregating tweets using pooling schemes based on author, burst score, hashtags and temporal aspects. Author topic model has been extended to include the countries of tweets (Morchid et al. 2015) for predicting the country of non-located tweets. Wang et al. $(2014,2016)$ suggested a 'hashtag graph based topic model' and retrieved relations between tweets and hashtags from the graph for finding semantically related words with and without co-occurrence. Lozano et al. (2017) presented a geo-aware topic model for identifying locations from the tweets. Topic models for examining topics over time in tweets must integrate the topic's distribution over words, hashtags and locations, to track the influence of co-occurrence as well as location contexts. A dynamic mechanism can be incorporated into the topic model to study the topic drift in tweets, that are partitioned into different snapshots over time.

\subsection{Tracking Dynamic Topics}

Traditional time series models are suitable for continuous data, whereas topic models are defined for identifying the group wise patterns in the corpus. Wang \& McCallum (2006) described a continuous time model where the topic is distributed over words as well as timestamps. Blei \& Lafferty (2006) employed the variational Kalman filtering and wavelet regression for posterior inference of parameters of a dynamic topic model for the topic evolution of sequentially organized corpus. The dynamic topic model can be scaled to a large number of topics (Bhadury et al. 2016) using stochastic gradient Langevin dynamics with no restricting assumptions and faster posterior inference. The dynamic model designed for topics in tweets should be able to process the varying dynamic behavior of hashtags and geotags in addition to words. 


\subsection{Topical Word Embedding}

Topic models can be enriched using a WE model. Li et al. (2016) employed Von-Mises-Fisher mixture model for topic representation and sampled word embedding for each topic by integrating the topic model and word embedding model. Qiang et al. (2017) extended LDA with Markov random field model and used WE from 'glove2vec' to get latent topical embedding for short texts. Xun et al. (2016) trained the WE model with Wikipedia, extracted topics using Gaussian LDA and modeled each short text as single Gaussian topic instead of mixture of topics. Jiang et al. (2016) constructed unified framework of topic and word embedding models based on which words were either generated using multinomial distribution or derived from the topic and word embedding. Niu et al. (2015) and Liu et al. (2015) modified WE model for learning topic representation called 'topic 2 vec' for representing words and topics in the same semantic space. Simple distance measures were applied to find nearest words of each topic instead of probabilities. Tags contexts can be used to enhance TWE, for estimating drift in tweets such that topics and words have different embedding vectors based on their association with different hashtags and geolocations.

The model planned in this paper, describes a topic model LDA_Tags with hashtags and geotags as topic indicators, employs discrete time dynamic topic model based on SGLD with varying mini-batch sizes to incorporate the asynchronous dynamic behavior of words, hashtags and geotags, and defines a topical word embedding model which jointly learns hashtag and geolocation contexts for effective detection of location based topic drift in tweets.

\section{JOINT TOPICAL WORD EMBEDDING MODEL WITH HASHTAG AND GEOTAG CONTEXTS}

In most of the literature (Wang \& McCallum 2006; Knights et al. 2009; Cataldi et al. 2010; Liu et al. 2013), LDA was used for analyzing the change in the distribution of topic over words at different time intervals. The contextual variation with the word was studied for topic drift. Limited work was done with other attributes like hashtags (Alam et al. 2017) and location (Bernabe-Moreno et al. 2015). But in the realtime scenario, geotags also have significant impact on topic drift. 
The proposed system (Figure 1) describes LDA for tags called LDA_Tags to integrate hashtags and geotags as topic indicators. Posterior inference of discrete time dynamics of LDA_Tags is performed using the parallelizable Gibbs sampling with stochastic gradient Langevin dynamics with varying mini-batch sizes (SGLD_vm) for accommodating the asynchronous distribution of words, hashtags and geotags. The mini-batch size of words, hashtags and geotags for each time slot is computed based on their distribution in the previous time interval to ensure that varying mini-batches correlates with varying dynamic behavior of words and tags. This is completely different from the fixed mini-batch size based on average length of documents (Bhadury et al. 2016) and from the step size decreasing to zero (Welling \& Teh 2011). Finally, the (word, topic) pairs and their posterior probabilities obtained from dynamic topic model are used to represent TWE by a group of context words, set of hashtags related to the contextual semantics and a collection of geotags connected with the location based semantics. The joint learning of these topic representations yields, tags context based TWE vectors. The change in the topical clusters of these embeddings (Niu et al. 2015) over the time will provide the evidence of topic drift. The mean squared deviation (MSD) of the se embeddings (Yang \& Tonnat 2017) with previous snapshots is used as a measure of topic drift.

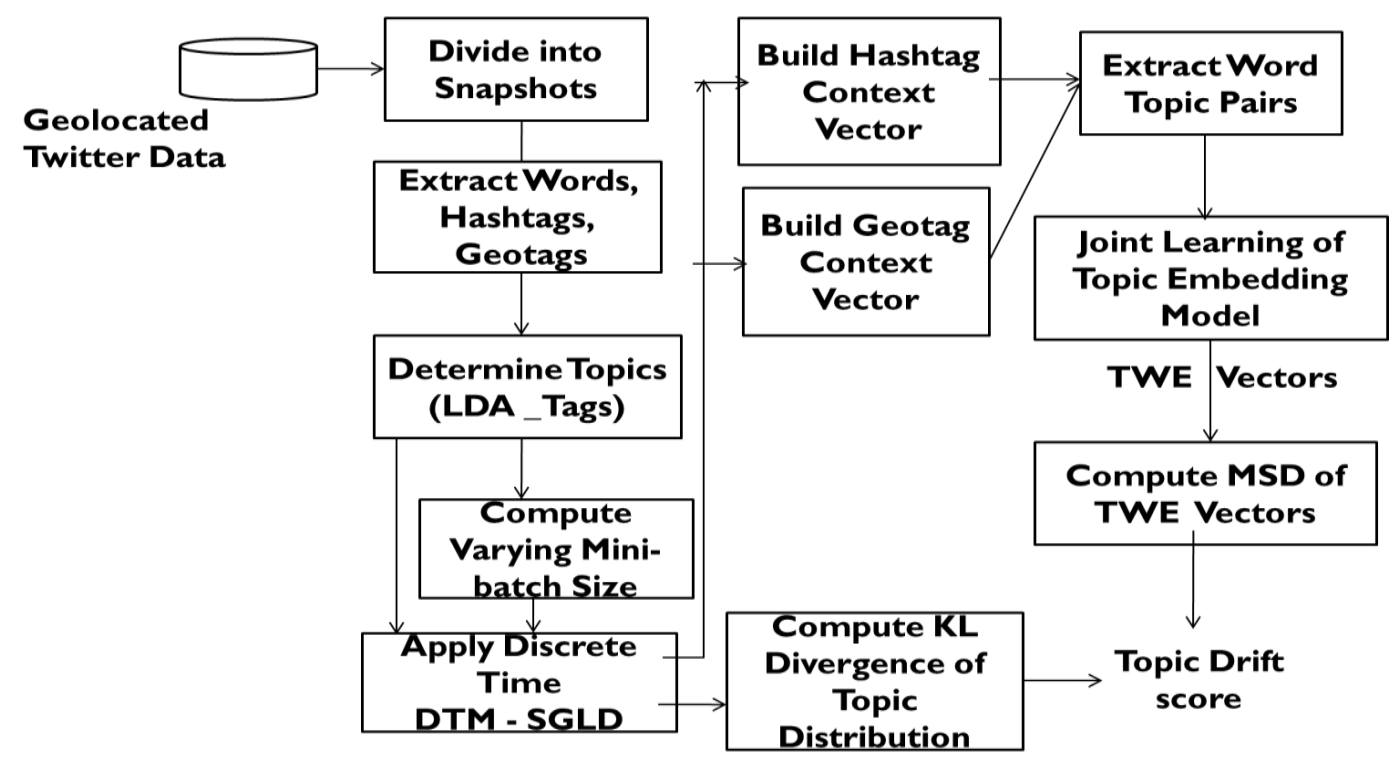

Figure 1. Joint Topical Word Embedding Model 


\subsection{Topic Model with Hashtag and Geotag Attributes}

Hashtags describe contextual semantics and are distributed over topics. Words cooccurring with certain hashtags in different tweets are semantically related. Similarly, tweets from a particular geographic region are having semantically related content and it is a fact that geolocations have an impact on the topic (Lozano et al. 2017) as well as topic drift in addition to words and hashtags. LDA_Tags differs from the topic model defined by Alam et al. (2017) and Wang et al. (2014) with geotags context and discrete time attribute. The time stamp is same for all data at a particular snapshot. It also differs from the author topic model (Rosen-Zvi et al. 2004) in grouping the tweets based on hashtags and the geolocation instead of author. No predefined label set is used as in labeled LDA (Mehrotra et al. 2013). Tag weighted LDA described by Li et al. (2013) is different from LDA_Tags by defining (authors, keywords) as tags and assigning tag weights for multi-tagged semi structured documents.

\subsubsection{LDA}

Text documents are modeled using LDA (Blei et al. 2003) based on the assumption 'conditionally independent and identically distributed' where, documents D is represented as a mixture of topics using Dirichlet distribution $(\theta)$ and topic ' $z$ ' is distributed over words (w) based on the multinomial distribution ( $\Phi$ ) (Figure 2). Topic models enable new types of semantic representation of words, allowing them to be present under more than one topic based on different senses (Griffiths et al. 2007). Topic proportion $\theta_{d}$ is sampled per each document, whereas the topic $z_{d w}$ and word $w_{d w}$ are sampled per each word ' $w$ ' in the document ' $d$ ' (Algorithm 1).

The probability distribution of words ' $w$ ' in document ' $\mathrm{D}$ ' sampled under the topic ' $z$ ' is described as

$p(w \mid D)=p(w \mid z) * p(z \mid D)$

Gibbs sampling is suitable for generating posterior probabilities by sampling variables from the conditional distribution. With LDA, the joint probability of topic proportion ' $\theta$ ', set of ' $k$ ' topics ' $z$ ' and a set of ' $N_{d}$ ' words are given (Blei et al. 2003) as

$p(\theta, z, w \mid \alpha, \beta)=p(\theta \mid \alpha) \prod_{d w=1}^{N_{d}} p\left(z_{d w} \mid \theta\right) p\left(w_{d w} \mid z_{d w}, \beta\right)$ 


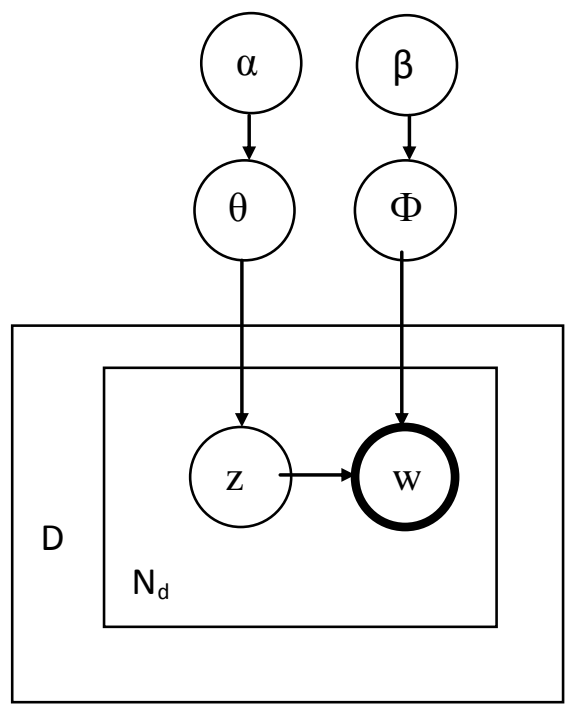

Figure 2. LDA Model

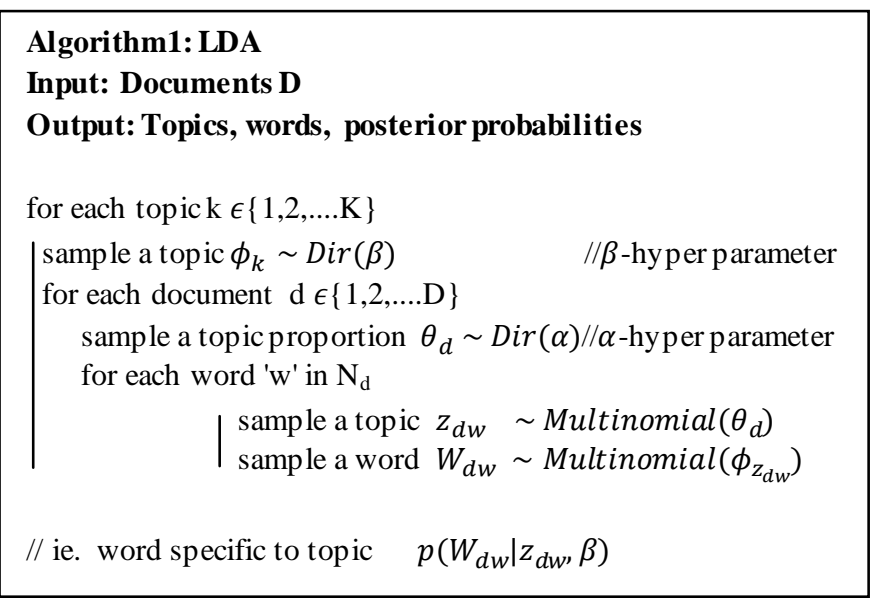

Algorithm 1. LDA

LDA has been extended in a variety of ways based on the type of application. Ramage et al. (2009) described a labeled LDA that represents multi-labeled corpora as a list of words \& labels and assigns labels for topics only from the given label set. They used labeled LDA for characterizing micro blogs (Ramage et al. 2010) by incorporating tweet level labels like hashtags, replies and emoticons which might be useful for classification and link prediction (Quercia et al. 2012). Multi contextual LDA presented by Tang et al. (2013) is a generalized contextual model applicable to any type of data having different contexts. They applied the model to Twitter data for the user and hashtag contexts. Twitter LDA (Zhao et al. 2011) treats each tweet as a document and identifies the word distribution for topics and background words. However, other contents like hashtags, urls and mentions are not considered. Hashtags are labels intended for the content and are useful to find related content on the same social media platform. Wang et al. $(2014,2016)$ focused on the topic model based on 'hashtag graph' for connecting the semantically related words. However, hashtags and geotags are required to be added into the topic model in addition to words.

\subsubsection{LDA_Tags}

The proposed LDA_Tags differs from the topic model described by Rosa et al. (2011) by including geotags for location specific context in addition to hashtags. The graphical representation of LDA with hashtags and geotags (Figure 3) as topic indicators describe show the 
context of both hashtags and geotags can be incorporated into the topic model for the analysis of topic drift in the media text. Hashtags ' $y$ ' and geotags ' $x$ ' are distributed over topics based on the Dirichlet distribution $(\theta)$ and topic ' $z$ ' has multinomial distribution $(\Phi)$ over words.

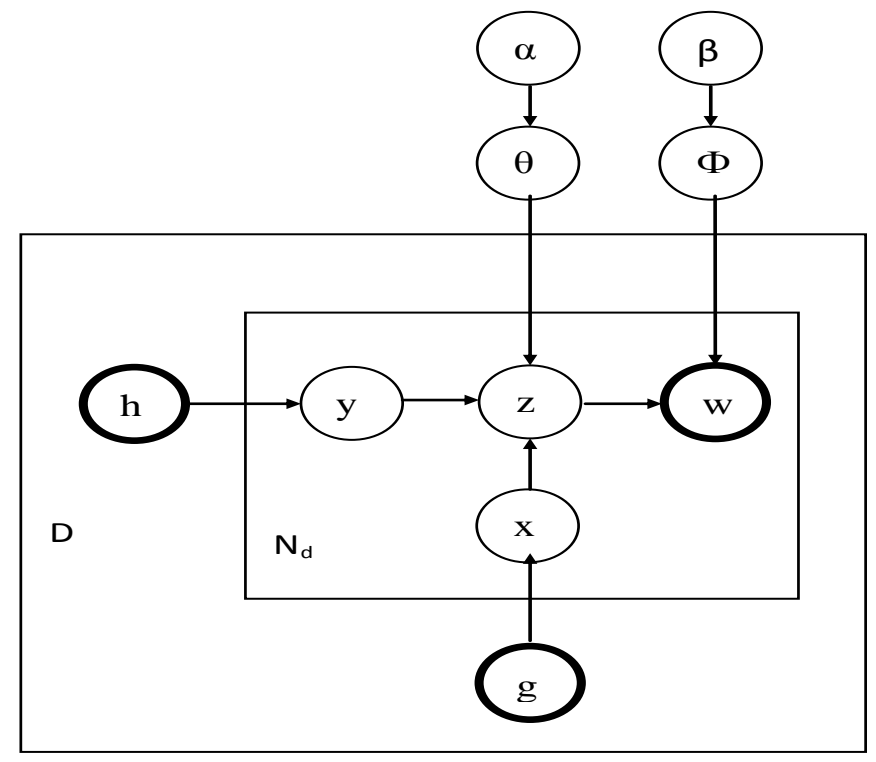

Figure 3. LDA_Tags

Now the document is modeled as a mixture of topics $(z)$ and the topics as a mixture of distributions over the words $(w)$, hashtags $\left(h_{v}\right)$ and geotags $\left(g_{v}\right)$. With LDA_Tags, the probability distribution of word ' $w$ ', hashtag ' $y$ ' and geotag ' $x$ ' in document ' $D$ ' sampled under the topic ' $z$ ' are described as done by Blei et al. (2003) for words.

$$
\begin{aligned}
& p(w, \boldsymbol{y}, \boldsymbol{x} \mid D)=p(w, \boldsymbol{y}, \boldsymbol{x} \mid z) * p(z \mid D) \\
& p(w \mid D)=\sum_{z} p(z) \prod_{d w=1}^{N_{d}} p\left(w_{d w} \mid z\right) \\
& p(y \mid D)=\sum_{z} p(z) \prod_{d w=1}^{N_{d}} p\left(y_{d w} \mid z\right) \\
& p(x \mid D)=\sum_{z} p(z) \prod_{d w=1}^{N_{d}} p\left(x_{d w} \mid z\right)
\end{aligned}
$$




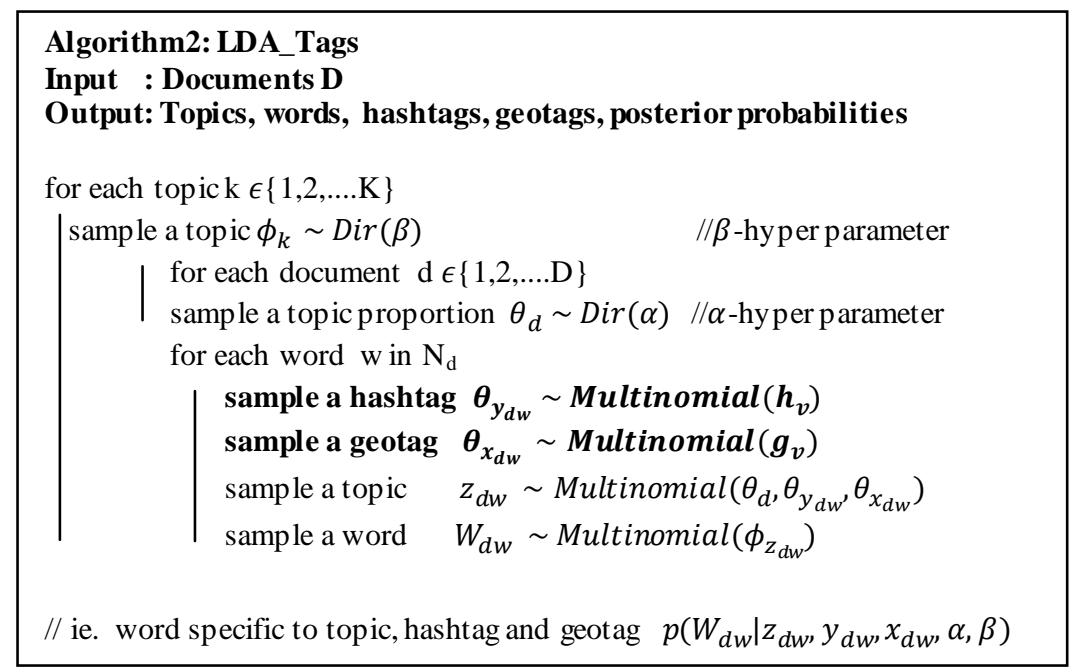

\section{Algorithm 2. LDA_Tags}

Vocabularies of words $(W)$, hashtags $\left(h_{v}\right)$, geotags $\left(g_{v}\right)$ and hyper parameters $(\alpha, \beta)$ define the topic model. Topic proportion $\theta_{d}$ is sampled per each document, whereas hashtag $\theta_{y_{d w}}$, geotag $\theta_{x_{d w}}$, topic $z_{d w}$ and word $w_{d w}$ are sampled per each word ' $w$ ' in the document ' $d$ ' (Algorithm 2). Now, the joint probability of the topic proportion $(\theta)$ with a set of ' $k$ ' topics, a set of $\mathrm{N}_{\mathrm{d}}$ words, hashtags \& geotags for the given hyper parameters $(\alpha, \beta)$ is defined by modifying the above equation (2) as,

$p(\theta, z, w \mid \alpha, \beta)=p\left(\theta_{d} \mid \alpha\right) \prod_{d w=1}^{N_{d}} p\left(z_{d w} \mid \theta_{d}, y_{d w}, x_{d w}\right) p\left(w_{d w} \mid z_{d w}, \beta\right)$

Where, $\mathrm{h}_{\mathrm{v}}$ - hashtag vocabulary, $\mathrm{g}_{\mathrm{v}}$ - geotag vocabulary, $y_{d w^{-}}$hashtag distribution, $x_{d w}$ - geotag distribution.

In Twitter data, hashtags have faster dynamics compared to that of words and geotags. New hashtags are created every day and their distributions are unpredictable. Hence, varying granularities are to be processed for examining the distribution of words, hashtags and geotags.

\subsection{Topic Dynamics with Hashtag and Geotag Contexts}

The proposed dynamic topic model is based on stochastic process that represents the system with random variables whose probability distributions are randomly changing over time. The parallelizable stochastic gradient Langevin dynamics (SGLD) model, which is suitable for the bigdata is redefined for inferring dynamic parameters of the LDA_Tags. Stochastic gradient Langevin dynamics with varying mini-batch sizes (SGLD_vm) is based on discrete time and is different from the continuous time dynamic topic models (Alam et al. 2014; Bhadury et al. 
2016). Varying mini-batch sizes are computed based on the varying distribution of words, hashtags and geotags over snapshots instead of random mini-batch selection by Welling \& Teh (2011). It is different from the dynamic models of Welling \& Teh (2011) and Bhadury et al. (2016) with the inclusion of asynchronous distribution of various attributes of tweets.

Let ' $D_{t}$ ' represents data at discrete time slot ' $t$ '. Dynamic model which chains the parameters in Markovian structure is defined using normal distribution with mean ' $\alpha_{t}$ ' and variance ' $\sigma$ ' (Bhadury et al. 2016).

$\alpha_{t} \sim \aleph\left(\alpha_{t} \mid \alpha_{t-1}, \sigma^{2} I\right)$

$\phi_{k t} \sim \aleph\left(\phi_{k t} \mid \phi_{k t-1}, \beta^{2} I\right) k \in K$

The proposed dynamic topic model is described using stochastic gradient Langevin dynamics (SGLD), an incremental gradient descent for minimizing an objective function which runs through a subset of samples. The SGLD parameter for ' $\theta$ ' at $\mathrm{t}^{\text {th }}$ iteration is given (Welling \& Teh 2011) as

$\left.\theta_{t+1}-\theta_{t}=\nabla \theta_{t}=\frac{\epsilon_{t}}{2}\left(\nabla \log \mathrm{p}\left(\theta_{\mathrm{t}}\right)+\frac{\mathrm{N}}{\mathrm{m}} \sum_{i=1}^{m} \nabla \log p\left(x_{t}^{i} \mid \theta\right)\right)+\xi_{t}\right)$

where, $\quad$ SG noise $=\frac{N}{m} \sum_{i=1}^{m} \nabla \log p\left(x_{t}^{i} \mid \theta\right) \quad$ LD noise $=\xi_{t} \sim \aleph\left(\xi_{t} \mid 0, \epsilon_{t}\right)$

$N$ - set of data items, $\mathrm{m}$ - mini-batch size, $x_{t}^{i}$ - mini-batch data items, $\epsilon_{t}$ - step size or learning rate

The dynamic parameters are inferred using methods like 'Gibbs sampling' which resamples each random variable iteratively given the remaining variables. However, different mini-batch sizes of words, hashtags and geotags for different snapshots are required to be computed.

\subsubsection{Varying Mini-batch Size}

The dynamic topic model for LDA_Tags must handle the difference in the rate of change distribution in words and tags. Random and independent selection of mini-batch sizes (Welling \& Teh 2011) is not appropriate for posterior inference of LDA_Tags and topic drift estimation. Larger mini-batch size may not be able to track contextual change with words and hashtags 
effectively and smaller mini-batch size may lead to unnecessary processing of location specific distribution. An increase in mini-batch size leads to decrease in convergence rate and reduces the communication cost ( $\mathrm{Li}$ et al. 2014). Hence, both small and large mini-batch sizes can be optimally initialized and can be varied based on distribution in previous snapshots for learning change in the posterior distribution. Mini-batch sizes for words $\left(m_{w}^{t+1}\right)$, hashtags $\left(m_{h}^{t+1}\right)$ and geotags $\left(m_{g}^{t+1}\right)$ to be replaced for ' $m$ ' in equation (10) can be computed as

$m_{w}^{t+1}=\frac{1}{N_{d}} \sum_{d w=1}^{N_{d}} p\left(w_{d w} \mid z, t\right)$

$m_{h}^{t+1}=\frac{1}{N_{d}} \sum_{d w=1}^{N_{d}} p\left(y_{d w} \mid z, t\right)$

$m_{g}^{t+1}=\frac{1}{N_{d}} \sum_{d w=1}^{N_{d}} p\left(x_{d w} \mid z, t\right)$

Dynamic topic model parameters for distribution of words and tags cannot be integrated and must be computed separately.

The discrete time dynamic model for LDA_Tags (Figure 4) links the topic parameters $(\alpha, \beta, \theta)$ for the three snapshots $(\mathrm{t}-1, \mathrm{t}, \mathrm{t}+1)$ and their variance parameters $(\sigma, \psi, \varphi)$ in a Markovian structure.

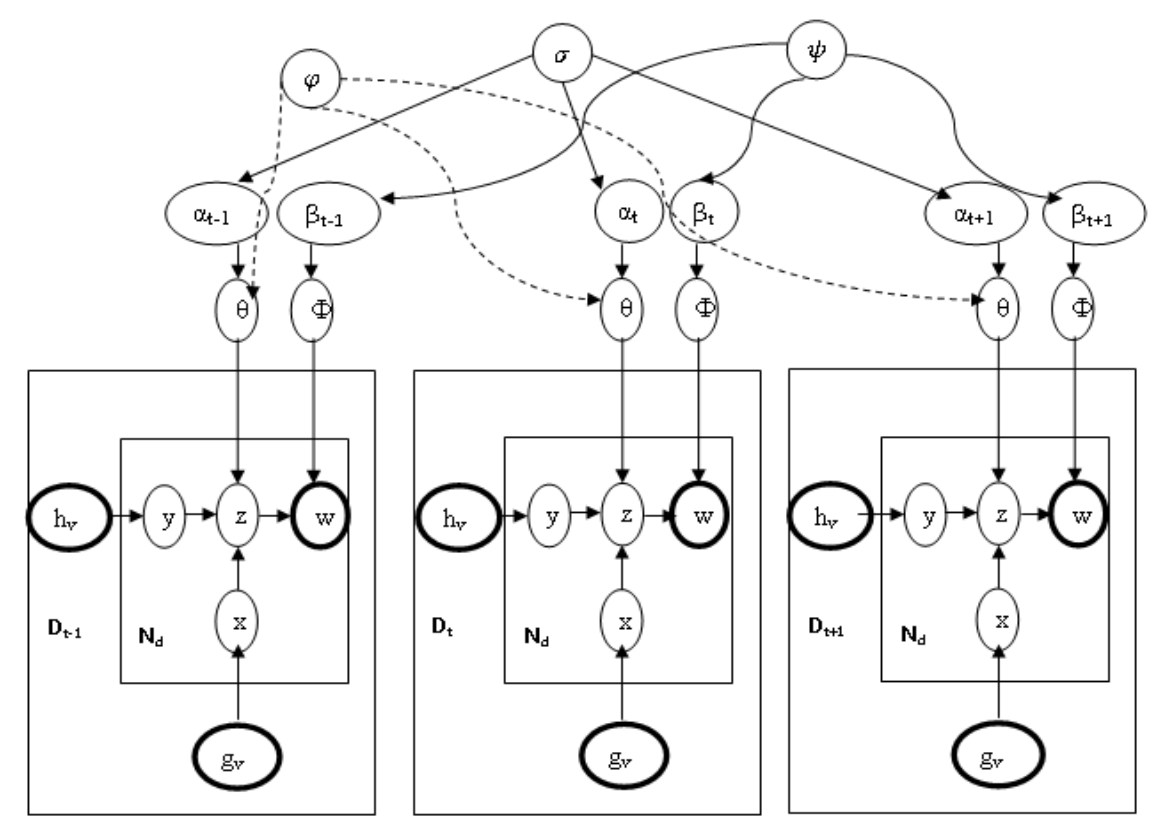

Figure 4. Topic Dynamics with Hashtag and Geotag Contexts 
Now, the dynamic parameters for SGLD with LDA_Tags can be derived as done by Bhadury et al. (2016) for LDA.

(i) Sampling parameter for mean at time ' $t$ ', $\alpha_{t}$

$$
\begin{gathered}
p\left(\alpha_{t} \mid M B\left(\alpha_{t}\right)\right) \propto \aleph\left(\alpha_{t} \mid \alpha_{t-1}, \sigma^{2} I\right) \aleph\left(\alpha_{t+1} \mid \alpha_{t}, \sigma^{2} I\right) \prod_{d t=1}^{D_{t}} \aleph\left(\theta_{d t} \mid \alpha_{t}, \psi^{2} I\right) \\
\theta_{d t} \sim \aleph\left(\theta_{d t} \mid \alpha_{t}, \psi^{2} I\right)
\end{gathered}
$$

(ii) Sampling parameter for topic-document proportion at time ' $t$ ', $\theta_{d t}$

$$
\begin{gathered}
p\left(\theta_{d t} \mid \alpha_{t}, z_{d t}\right) \propto \aleph\left(\alpha_{t} \mid \alpha_{t-1}, \varphi^{2} I\right) \aleph\left(\theta_{d t} \mid \alpha_{t}, \varphi^{2} I\right) \prod_{d w t=1}^{N_{d t}} \operatorname{Mult}\left(z_{d w t} \mid \pi\left(\theta_{d t}\right)\right) \\
\theta_{d t} \sim \aleph\left(\theta_{d t} \mid \alpha_{t}, \varphi^{2} I\right)
\end{gathered}
$$

Where $\pi(a)=b$ with each element $\quad b_{l}=\frac{\exp \left(a_{l}\right)}{\sum_{j} \exp \left(a_{j}\right)}$

(iii) Sampling parameter for hashtag-topic proportion at time ' $t$ ', $\theta_{y_{d t}}$

$$
\begin{gathered}
p\left(\theta_{y_{d t}} \mid \alpha_{t}, z_{d t}\right) \propto \aleph\left(\alpha_{t} \mid \alpha_{t-1}, \varphi^{2} I\right) \aleph\left(\theta_{y_{d t}} \mid \alpha_{t}, \varphi^{2} I\right) \prod_{d w t=1}^{N_{d t}} \operatorname{Mult}\left(z_{d w t} \mid \pi\left(\theta_{y_{d t}}\right)\right. \\
\theta_{y_{d t}} \sim \aleph\left(\theta_{y_{d t}} \mid \alpha_{t}, \varphi^{2} I\right)
\end{gathered}
$$

(iv) Sampling parameter for geotag-topic proportion at time ' $t$ ' $\theta_{x_{d t}}$

$$
\begin{gathered}
p\left(\theta_{x_{d t}} \mid \alpha_{t}, z_{d t}\right) \propto \aleph\left(\alpha_{t} \mid \alpha_{t-1}, \varphi^{2} I\right) \aleph\left(\theta_{x_{d t}} \mid \alpha_{t}, \varphi^{2} I\right) \prod_{d w t=1}^{N_{d t}} \operatorname{Mult}\left(z_{d w t} \mid \pi\left(\theta_{x_{d t}}\right)\right. \\
\theta_{x_{d t}} \sim \aleph\left(\theta_{x_{d t}} \mid \alpha_{t}, \varphi^{2} I\right)
\end{gathered}
$$

(v) Sampling parameter for a topic from ' $k$ ' at time ' $t$ ', $\phi_{k t}$

$$
p\left(\phi_{k t} \mid M B\left(\phi_{k t}\right)\right) \propto \aleph\left(\phi_{k t} \mid \phi_{k t-1}, \psi^{2} I\right) \aleph\left(\phi_{k t+1} \mid \phi_{k t}, \psi^{2} I\right) \prod_{d w t=1}^{N_{d t}} \operatorname{Mult}\left(w_{d w t} \mid \pi\left(\phi_{k t}\right)\right.
$$

(vi) Sampling topic ' $z$ ' for word at time ' $t$ ', $z_{d w t}$

The topic sampling, is given by collapsing topic-document proportion ( $\left.\theta_{d t}^{k}\right)$, hashtag-topic proportion $\left(\theta_{y_{d t}}^{k}\right)$, geotag-topic proportion $\left(\theta_{x_{d t}}^{k}\right)$ and the topic-word proportion $\left(\phi_{k t}^{w}\right)$. 
$p\left(z_{d w t}=k \mid \theta_{d t}^{k}, \theta_{y_{d t}}^{h_{v}}, \theta_{x_{d t^{\prime}}}^{g_{v}} \phi_{k t}^{w}\right) \propto \exp \left(\theta_{d t}^{k}\right) \exp \left(\theta_{y_{d t}}^{k}\right) \exp \left(\theta_{x_{d t}}^{k}\right) \exp \left(\phi_{k t}^{w}\right)$

\subsubsection{Kullback-Leibler Divergence of Topic}

Topic drift can be described by Kullback-Leibler (KL) divergence (Cai et al. 2014) of distribution of a topic $z_{1}^{t}$ over words ' $w$ ', conditioned on hashtag ' $y$ ' and geotag ' $x$ ' over time as

$D_{K L}\left(z_{1}^{t} \| z_{1}^{t+1}\right)=\sum_{w=1}^{W} P\left(z_{1}^{t} \mid w, y, x\right) \ln \frac{P\left(z_{1}^{t} \mid w, y, x\right)}{P\left(z_{1}^{t+1} \mid w, y, x\right)}$

If KL divergence of a topic is high during a particular period, it denotes a drift in the topic during that interval.

\subsection{Topical Word Embedding with Hasgtag and Geotag Contexts}

Topics can be better associated with tags when represented as a vector and the representation is learnt with an embedding model. Additional knowledge can be incorporated into TWE by replacing 1-of- v vector with a two dimensional vector as done by Bian et al. (2014) where each row was added to characterize a particular attribute by incorporating lexical and morphological features. The topic representation conditioned on hashtags (Figure 5a) and geotags (Figure 5b) differ from that of Bian et al. (2014) by denoting the topical word as a group wise distribution of words with the co-occurring tags and location contexts. Top 100 hashtags with the highest frequency $(\geq 50)$ in the tweet corpus and top 100 geotags from which more tweets $(\geq 100)$ have been sent, are selected. Topics, words and their probability distributions are generated from the topic model with tags context (LDA_Tags). Entries of hashtag context vector (HCV) (Figure 5a) represents the association of topic 'zh' with the context words $\left(\mathrm{wc}_{1}, \mathrm{wc}_{2}, \mathrm{wc}_{3}, \mathrm{wc}_{4} \ldots . . . \mathrm{wc}_{\mathrm{h}}\right)$ and hashtags $\left(\mathrm{y}_{1}, \mathrm{y}_{2}, \mathrm{y}_{3}, \ldots, \mathrm{y}_{100}\right)$ whereas, geotag context vector (GCV) (Figure 5b) represents the association of topic 'zg' with the context words $\left(\mathrm{wc}_{1}, \mathrm{wc}_{2}, \mathrm{wc}_{3}, \mathrm{wc}_{4} \ldots . . \mathrm{wc}_{\mathrm{g}}\right)$ and geotags $\left(\mathrm{x}_{1}, \mathrm{x}_{2}, \mathrm{x}_{3}, \ldots, \mathrm{x}_{100}\right)$. The set of context words related to topic ' $\mathrm{zg}$ ' in GCV may be different from words in HCV. Same topic has different vector representations when associated with different hashtags or geolocations. 


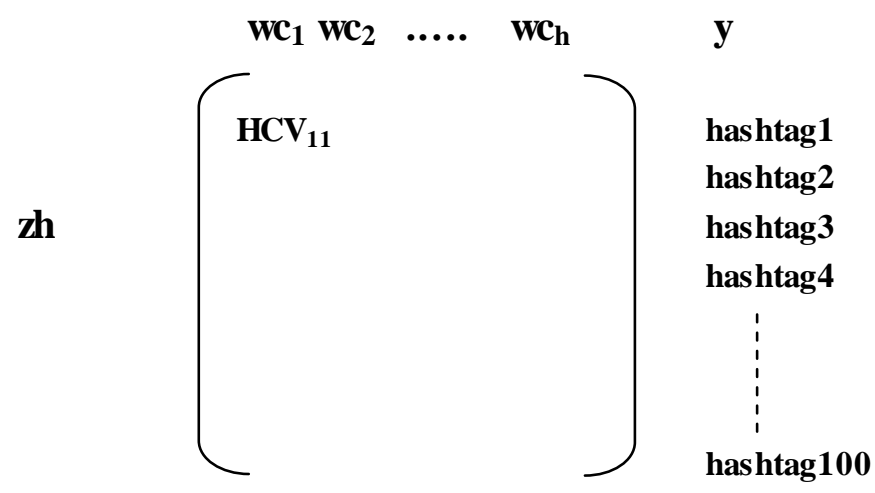

(a) Hashtag Context Vector

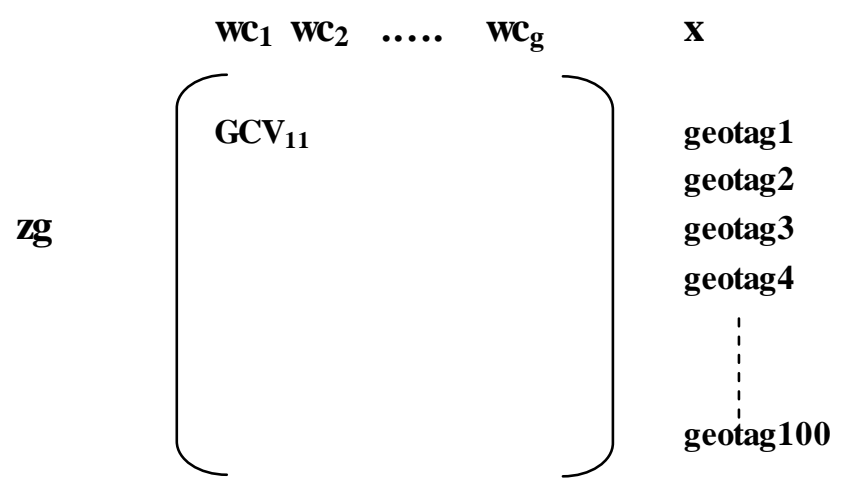

\section{(b) Geotag Context Vector}

\section{Figure 5. Topical Word Embedding}

Each entry $\left(\mathrm{HCV}_{11}\right)$ in the hashtag context vector (Figure 5a) is computed as, $p\left(z h \mid w c_{1}, y_{1}\right) *$ $h f_{11}$, where ' $h f_{11}$ ' represents the frequency of occurrence of word ' $w c_{1}$ ' with the given hashtag ' $y_{1}$ '. Similarly, the geotag context vector entry (Figure 5b) is described as, $\mathrm{p}\left(z g \mid w c_{1}, x_{1}\right) * g f_{11}$, where ' $g f_{11}$ ' equals the number of tweets with word ' $w c_{1}$ ' posted from the geolocation ' $x_{1}$ '.

Each (word, topic) pair is associated with a 200 dimensional vector obtained by concatenating columns of HCV and GCV. The topical word embedding model is trained using these (word, topic) pairs to yield topic vectors with tags context. Learning is done using the average of log likelihood of the posterior probabilities. The equations are derived based on the TWE model by Liu et al. (2015) as 
$L_{\text {hash }}=\frac{1}{W}\left(\sum_{i=1}^{W} \sum_{1<j<i-1}^{i+1<j<h} \log P\left(w c_{j} \mid z h_{i}\right)+\log P\left(w c_{j} \mid y_{i}\right)\right)$

$L_{\text {geo }}=\frac{1}{W}\left(\sum_{i=1}^{W} \sum_{1<j<i-1}^{i+1<j<g} \log P\left(w c_{j} \mid z g_{i}\right)+\log P\left(w c_{j} \mid x_{i}\right)\right)$

The WE model predicts the context words for the given word, whereas, TWE predicts the context words for the given word and the topic. However, the proposed TWE model is able to predict the context words for the given (word, topic) pair conditioned on hashtag and geotag contexts (Equation 25). Both HCV and GCV can be jointly learned as performed by Niu et al. (2016).

$L_{\text {hash\&geo }}=\frac{1}{w}\left(\sum_{i=1}^{W} \sum_{1<j<i-1}^{i+1<j<C} \log P\left(w c_{j} \mid z h_{i}\right)+\log P\left(w c_{j} \mid z g_{i}\right)+\log P\left(w c_{j} \mid y_{i}\right)+\log P\left(w c_{j} \mid x_{i}\right)+\log P\left(w c_{j} \mid t\right)\right)$

The joint learning of topical embedding using both hashtag and geotag context vectors is described as below (Figure 6).

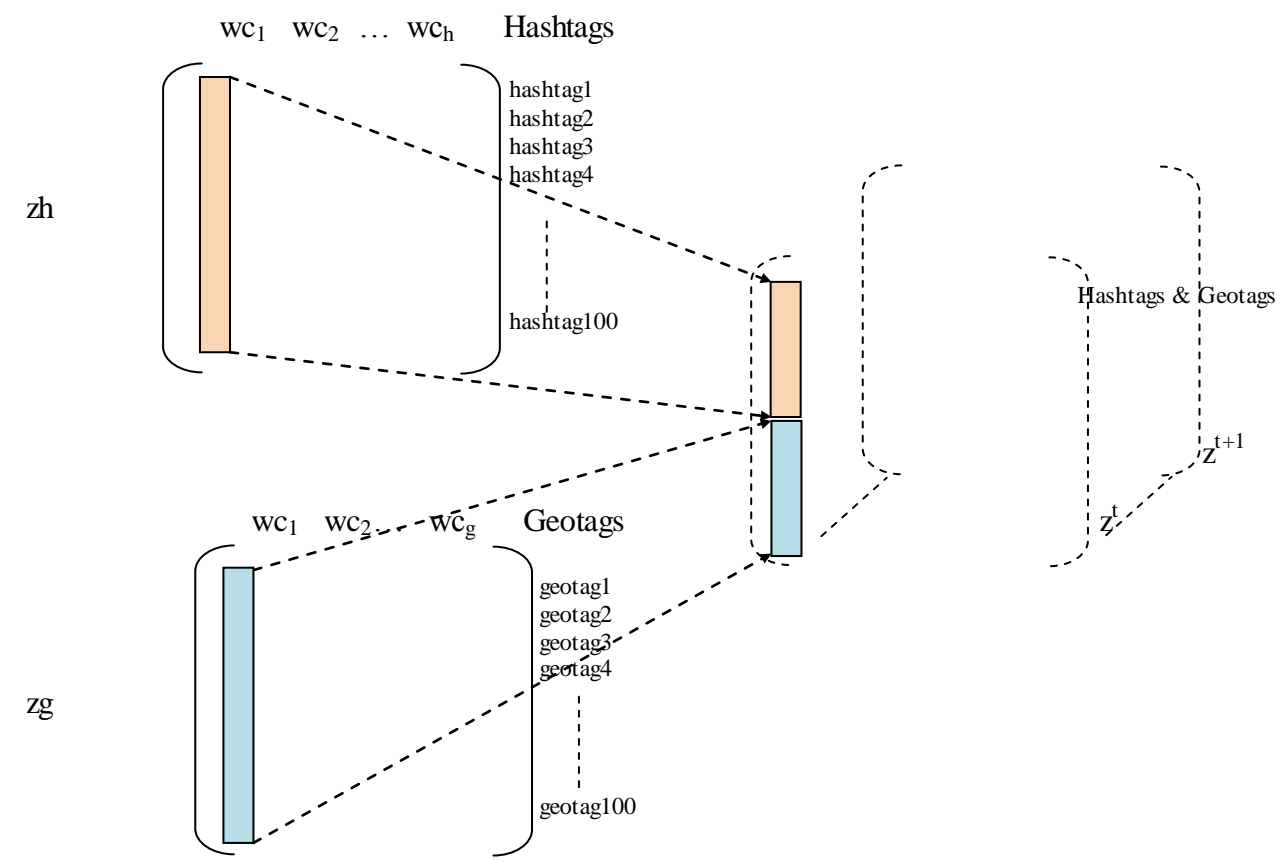

Figure 6. Joint Learning of Hashtag and Geotag Contexts

Once the model is trained with the two sets of word_topic pairs $\left[\left(\left\langle\mathrm{wc}_{1} \_\mathrm{zh}\right\rangle^{\mathrm{t}},\left\langle\mathrm{wc}_{2} \mathrm{zh}\right\rangle^{\mathrm{t}}, \ldots\right.\right.$, $\left.\left.\left\langle\mathrm{wc}_{\mathrm{h} \_} \mathrm{zh}\right\rangle^{\mathrm{t}}\right)\right]$ and $\left[\left(\left\langle\mathrm{wc}_{1} \_\mathrm{zg}\right\rangle^{\mathrm{t}},\left\langle\mathrm{wc}_{2} \mathrm{zg}\right\rangle^{\mathrm{t}}, \ldots\left\langle\mathrm{wc}_{\mathrm{g} \_} \mathrm{zg}\right\rangle^{\mathrm{t}}\right)\right]$ inferred from the topic model, it is capable of predicting the word_topic pairs specific to the <word_topic> pair that are contextually 
related to a set of hashtags and geotags at time ' $t$ '. Learning can be done incrementally with word_topic pairs from different snapshots to obtain TWE vectors $\left(w c_{-} z c^{v}\right)$ of word_topic pairs for estimating the drift in the topics.

The softmax function is described (Liu et al. 2015) as

$P\left(\left\langle w c_{-} z c\right\rangle \mid\left\langle w_{i-} Z_{i}\right\rangle\right)=\frac{\exp \left(w c_{-} z c^{v} \cdot w_{i-} z_{i}{ }^{v}\right)}{\sum_{w c_{-} z c \in(C, Z)} \exp \left(w c_{-} z c^{v} \cdot w_{i-} z_{i}{ }^{v}\right)}$

\subsubsection{Topic Drift}

Topics at a given period may evolve from the topics at the previous time interval. The topic drift is detected by estimating the mean squared deviation (MSD) of TWE vectors obtained for different snapshots of tweet corpus, as done by Yang \& Donnat (2017) for measuring word meaning change. Let $\left(V_{z}^{1}, V_{z}^{2}, \ldots V_{z}^{t-1}\right)$ represents the topical word embedding vectors of topic ' $z$ ' from 1 to $\mathrm{t}-1$ intervals. MSD of $V_{z}^{t}$ from these values will give the accurate measure of topic drift.

$\operatorname{MSD}\left(V_{z}^{t},\left(V_{z}^{1}, V_{z}^{2}, \ldots V_{z}^{t-1}\right)\right)=\left(V_{z}^{t}-\operatorname{mean}\left(V_{z}^{1}, V_{z}^{2}, \ldots V_{z}^{t-1}\right)\right)^{2}$

\section{EXPERIMENTAL SETUP AND EVALUATION}

\subsection{Dataset}

Chennai and UK geolocated tweets are used for investigating topic drift. A new Twitter application is created for getting an access token and consumer secret key. The geolocated tweets for 'Chennai' (latitude -13.067439, longitude-13.067439) and for keyword '\#chennai' are downloaded using API ${ }^{2}$ for three intervals (15.01.17-22.01.17), (24.05.17-31.05.17) and (20.08.17-27.08.17) (Table 1). Data obtained in JSON format is converted to CSV format and is preprocessed for creating a tweet corpus with vocabularies of words, hashtags and geolocations. The process is repeated for each time slot. UK-Geolocated dataset ${ }^{3}$ comprises of 1,69,033 tweets extracted from (2016-04-14 09:44) to (2016-04-21 08:30) using the keyword (-x_UK_X filter:nativeretweets geocode:55.378051,-3.43597299999999,750km). The dataset is split into seven snapshots from 14.04.16 to 21.04.16 (Table 1) and is preprocessed.

\footnotetext{
${ }^{2}$ https://dev.twitter.com/docs/streaming-api

${ }^{3}$ http://followthehashtag.com/datasets/170000-uk-geolocated-tweets-free-twitter-dataset/
} 
Table 1. Dataset

\begin{tabular}{lccll}
\hline Time Interval & $\begin{array}{l}\text { No of } \\
\text { Tweets }\end{array}$ & $\begin{array}{l}\text { No of } \\
\text { Words }\end{array}$ & $\begin{array}{l}\text { No of } \\
\text { Hashtags }\end{array}$ & $\begin{array}{l}\text { No of } \\
\text { Geotags }\end{array}$ \\
\hline & Chennai-Geolocated Tweet & & \\
\hline$(15.01 .17-22.01 .17)$ & 21968 & 5,969 & 893 & 1,350 \\
\hline$(24.05 .17-31.05 .17)$ & 22,441 & 10,048 & 2,644 & 2,573 \\
\hline$(20.08 .17-27.08 .17)$ & 27,399 & 6,359 & 1163 & 1,257 \\
\hline \multicolumn{5}{l}{ UK-Geolocated Tweet } \\
\hline 14.04 .2016 & 16,699 & 27,216 & 12,052 & 7870 \\
\hline 15.04 .2016 & 21,852 & 35819 & 18,888 & 6452 \\
\hline 16.04 .2016 & 31,451 & 44116 & 25,596 & 5136 \\
\hline 17.04 .2016 & 27,136 & 38900 & 20,133 & 6384 \\
\hline 18.04 .2016 & 20,384 & 31314 & 14,895 & 5707 \\
\hline 19.04 .2016 & 21,707 & 33031 & 16,569 & 6177 \\
\hline 20.04 .2016 & 24,177 & 36581 & 18,960 & 6629 \\
\hline
\end{tabular}

\subsection{Topic Model - Results}

Topics with posterior probabilities of words, hashtags and geotags are generated using LDA_Tags in combination with SGLD_vm. The dynamic topic model is initialized with learning rate of 0.018 and mini-batch sizes of 250 (words), 200 (hashtags), 300 (geolocations). The values for subsequent snapshots are changed based on the average of probabilities of previous snapshots. The topmost ten words, hashtags and geolocations (Table 2) for five topics show how the topics are distributed based on the location Chennai during January 2017. The trending topics identified during three snapshots are,

i) January_2017 -('college', 'jallikattu', 'actor', 'gonna','human chain')

ii) May_2017 - ('baahubali', 'beefban', 'Ilayaraja', 'chennaisilks' ,'vivegam')

iii) August_2017 - ('arrahman','mersal teaser','2.0', 'Ganesh Chaturthi', 'vivegam review') 
Table 2. Topics - Chennai Data (January_2017)

\begin{tabular}{|c|c|c|c|c|}
\hline Topic1 & Topic2 & Topic3 & Topic4 & Topic5 \\
\hline \multicolumn{5}{|c|}{ Words } \\
\hline College & Jallikattu & Actor & Karthiksubbaraj & Rameshlaus \\
\hline Use & Justice & Vijay & Gonna & Chain \\
\hline Pls & I am & Sangam & Decision & Human \\
\hline Contact & Legal & Nadigar & Accepted & Huge \\
\hline Rest & Str & Sethupathy & Strong & Formed \\
\hline Presedential & Marina & Justiceforjallikattu & News & Professionals \\
\hline Quens & Justicefor & Team & Till & Youth \\
\hline Girls & Save & Says & Ticket & Rest \\
\hline Marrys & Culture & Deccan & Loud & Girls \\
\hline Queries & Sri & Chronicle & Tamil & Protesters \\
\hline \multicolumn{5}{|c|}{ Hashtags } \\
\hline \#banpeta & \#saveourculturejallikattu & \#peta & \#peta & \#alanganallur \\
\hline \#amendpca & \#justiceforjallikattu & \#justiceforjallikattu & \#chennai & \#amendpca \\
\hline \#justiceforjallikattu & \#omr & \#vijay & \#jallikattu & \#jallikattu \\
\hline \multirow[t]{2}{*}{ \#jallikattuprotest } & \#jallikattuprotest & \#jallikattu & \#tamil & \#jallikattu \\
\hline & & & & Ordinance \\
\hline \#savejallikattu & \#amendpca & \#ilayathalapathy & \#modi & \#justiceforjallikattu \\
\hline \#justiceforjallikattu & \#jallikattu & \#wesupportjallikattu & \#tamilculture & $\#$ jallikattuprotest \\
\hline \#makkalmovement & \#coimbatore & \#rekla & \#tamilnadu & \#jallikattus logans \\
\hline \multirow[t]{2}{*}{ \#youthpower } & \#alanganallur & \#suriya & \#karthikeyasiva & \#jallikattuprotests \\
\hline & & & Senapathy & \\
\hline \#jallikattupermanent & \#jallikattuordinance & \#wedojallikattu & \#pakistani & \#marina \\
\hline \#jallikattu & \#jallikattuforpermanent & \#aiadmk & \#petaindia & \#protest \\
\hline \multicolumn{5}{|c|}{ Geotags } \\
\hline India & Chennai & chennai, India & Chennai & Chennai,india \\
\hline Chennai & chennai,india & Chennai & Chennai, india & Coimbatore \\
\hline Tamil & united & Lanka & Bangalore & Tamilnadu \\
\hline $\mathrm{Nadu}$ & Arab & Sri & Tamilnadu & Washington \\
\hline Nadu & emirates & Malaysia & Ambattur & Abroad \\
\hline Salem & Dubai & Dubai & Arabia & Hill \\
\hline Coimbatore & Uae & India & Kuwait & Usa \\
\hline Tamilnadu & Pondicherry & France & Chennai/Saudi & Chennai \\
\hline
\end{tabular}




\begin{tabular}{lllll}
\hline chennai,tamil & Tirupur & Tirunelveli & Bangalore & Fort \\
\hline Vellore & Tamilnadu & India & Dammam & Trichy \\
\hline
\end{tabular}

During January, the hot topics on Chennai based social media are 'jallikattu', 'chennai' and 'marina'. Jallikattu is a village based bull taming event played as part of Pongal celebrations in Tamil Nadu since 2500 years. Famous jallikattu locations are Alanganallur, Avaniapuram, Tiruvapur, Thammampatti, Palemedu, Sravayal, Kanduppatti, Venthanpatti and Pallavarayanpatty. But, due to the protest done by youngsters against the ban on 'jallikattu' during January_2017, the city based locations 'chennai', 'marina' and 'marinabeach' too are associated with 'jallikattu'. It is due to the cultural impact through social media that caused drift on topic 'jallikattu'.

Similarly, the topics ('beef ban', 'fire on chennai silks') during May_2017 and ('Ganesh Chaturthi', 'mersal teaser' and 'vivegam review') during August_2017 are not affected by drift (Table 3). Word_topic pairs for different snapshots are learnt (alpha - 0.025) incrementally with the proposed topical word embedding model (topic2 vec modified with LDA_Tags) to get topical embedding vectors over time.

Table 3. Topics - Chennai Data (May_2017)

\begin{tabular}{|c|c|c|c|c|}
\hline Topic1 & Topic2 & Topic3 & Topic4 & Topic5 \\
\hline \multicolumn{5}{|c|}{ Words } \\
\hline Says & Eat & Vandi & Chennai & Thalapathy \\
\hline Baahubali & Sooraj & Oodudhu & Fire & Beefban \\
\hline All & Who & Rotula & Silks & Vote \\
\hline Teaser & Banish & Ganaa & Now & Hange \\
\hline mithunraman & Media & Spiritual & Trending & Month \\
\hline Best & Beaten & Ilayaraja & Trends & Needed \\
\hline Shows & Madras & I am & Building & The \\
\hline cinemas & Kumar & Str & Google & Vivegam \\
\hline previous & Singh & Tune & Nagar & Schedule \\
\hline Wid & Other & Maestro & Tamil & September \\
\hline \multicolumn{5}{|c|}{ Hashtags } \\
\hline \#beefban & \#beefban & \#ilayaraja & \#chennaisilks & \#chennai \\
\hline
\end{tabular}




\begin{tabular}{|c|c|c|c|c|}
\hline \#chennai & \#iitmadras & \#beefban & \#tnagar & \#thalapathy61 \\
\hline \#3novices & \#beeffest & \#rottulavandioodudhu & \#chennai & \#sachinbilliondreams \\
\hline \#baahubali2 & \#kerala & \#spiritualganaa & \#chennaisilks fire & \#ilayathalapathy \\
\hline \#india & \#tamilnadu & \#aaa1d & \#maniratnam & \#europe \\
\hline \#news & \#beef & \#diravidanadu & \#fire & \#arrahman \\
\hline \#fashion & \#bjp & \#june2nd & \#fireaccident & \#vijay \\
\hline \#fridayfeeling & \#chennai & \#beef & \#tamilnadu & \#dangal \\
\hline \#3yearsofmodigovt & \#dravidanadu & \#is aignani & \#chennai & \#vivegam \\
\hline \#thondan & $\# g s t$ & \#maestro & \#chao & \#vijay61 \\
\hline \multicolumn{5}{|c|}{ Geotags } \\
\hline Chennai & Chennai & Tamil & Chennai & India \\
\hline tamilnadu & India & $\mathrm{Nadu}$ & Madras & Chennai \\
\hline egmore & Kerala & Chennai & Bangalore & Pondicherry \\
\hline nungambakkam & World & Trichy & Hyderabad & Coimbatore \\
\hline India & Cuddapah & Vijayawada & Earth & Pune \\
\hline nagercoil & Bengaluru & Coimbatore & France & Mumbai \\
\hline visakhapatnam & Gujarat & India & Chengalpattu & Hyderabad \\
\hline Chennai,india & India & Thoothukkudi & Vellore & Nellore \\
\hline chennai,tamil & Tirunelveli & Arumuganeri & Erode & Trivandrum \\
\hline Coimbatore & India & Tamilnadu & Pondicherry & Kerala \\
\hline
\end{tabular}

Table 4. Topics - Chennai Data (August_2017)

\begin{tabular}{|l|l|l|l|l|l|}
\hline \multicolumn{2}{|l|}{ Topic1 } & Topic2 & Topic3 & Topic4 & Topic5 \\
\hline \multicolumn{5}{|l|}{ Words } \\
\hline rahman & actorvijay & 2.0 & Ganesh & vivegam \\
\hline live & mersal & release & India & thala \\
\hline mersal & tweet & movie & Chaturthi & soon \\
\hline vijay & film & maniac & city & coming \\
\hline music & thalapathy & postponed & day & tickets \\
\hline thalapathy & opening & been & tweet & sudos \\
\hline hajj & teaser & respect & time & actor \\
\hline cinema & high & mutual & Chennai & Chennai \\
\hline pic & kerala & trailer & town & movie \\
\hline qkv & fans & film & speech & alltime \\
\hline
\end{tabular}




\begin{tabular}{|c|c|c|c|c|}
\hline \#arrahman & \#mersal & \#2point0 & \#ganeshchaturthi & \#vivegam \\
\hline \#mersal & \#arrahman & \#2.0 & \#pvsindhu & \#thala \\
\hline \#mersalmusiclive & \#mers almusiclive & \#rajinikanth & \#sainanehwal & \#ajith \\
\hline \#vijay & \#mersalarasan & \#akshaykumar & \#deshbachao & \#fanmade \\
\hline \#mersalaras an & \#tamilnadu & \#chennai & \#india & \#vedalam \\
\hline \#mers alalbum & \#vijay & \#shankar & \#banvaus & \#vivegamreview \\
\hline \#music & \#thalapathy & \#deraviolence & \#mannkibaat & \#tamiltalkies \\
\hline \#rahman & \#ts1100 & \#arrahman & \#mayweathervmcgregor & \#chennai \\
\hline \#arr & \#arr25 & \#yazhini & \#worldbadminton & \#mersal \\
\hline \#arr25 & \#mersalaudiolaunch & \#motherteresa & \#chao & \#vikramvedha \\
\hline \multicolumn{5}{|c|}{ Geotags } \\
\hline chennai & Chennai & Tamil & Chennai & India \\
\hline tamilnadu & India & Nadu & Madras & Chennai \\
\hline egmore & Kerala & Chennai & Bangalore & Pondicherry \\
\hline nungambakkam & World & Trichy & Hyderabad & Coimbatore \\
\hline India & Cuddapah & Vijayawada & Earth & Pune \\
\hline nagercoil & Bengaluru & Coimbatore & France & Mumbai \\
\hline visakhapatnam & Gujarat & India & Chengalpattu & Hyderabad \\
\hline Chennai,india & India & Thoothukkudi & Vellore & Nellore \\
\hline chennai,tamil & Tirunelveli & Arumuganeri & Erode & Trivandrum \\
\hline Coimbatore & India & Tamilnadu & Pondicherry & Kerala \\
\hline
\end{tabular}

\subsection{Evaluation}

The evaluation is performed for topic model, topic dynamic model, topical word embedding and topic drift. For topic models, the common parameter used for evaluation is perplexity (Blei et al. 2003). It gives a measure of how well a probability model predicts a sample or how much it is perplexed by a sample from the observed data. The lower the value of the perplexity, the model is better. The average perplexity (Bhadury et al. 2016) and relative perplexity (Knights et al. 2009) over the snapshots are computed for evaluating dynamic topic models. Topical clustering is compared for evaluating different topical embedding models (Niu et al. 2015). Topic drift is evaluated using KL divergence (Cai et al. 2014) and MSD (Yang \& Donnat 2017) measures.

\subsubsection{Topic Model Evaluation}


Based on perplexity computation by Blei et al. (2003), the perplexity for LDA_hash, LDA_geo and LDA_Tags are computed as

Perplexity $_{L D A} \quad=e^{-\frac{\sum_{d w=1}^{N} \log P\left(w_{d w}\right)}{\operatorname{count}(\text { words })}}$

Perplexity $_{L D A \_h a s h}=e^{-\left(\frac{\sum_{d w=1}^{N_{d}} \log P\left(w_{d w}\right)}{\text { count }(\text { words })}+\frac{\sum_{d w=1}^{N_{d}} \log P\left(y_{d w}\right)}{\operatorname{count}(\text { hashtags })}\right)}$

Perplexity $_{L D A_{\text {geo }}}=e^{-\left(\frac{\sum_{d w=1}^{N_{d}} \log P\left(w_{d w}\right)}{\operatorname{count}(\text { words })}+\frac{\sum_{d w=1}^{N_{d}} \log P\left(x_{d w}\right)}{\text { count (getota gs })}\right)}$

Perplexity ${ }_{L D A_{-} T a g s}=e^{-\left(\frac{\sum_{d w=1}^{N_{d}} \log P\left(w_{d w}\right)}{\operatorname{count}(\text { words })}+\frac{\sum_{d w=1}^{N_{d}} \log P\left(y_{d w}\right)}{\operatorname{count}(\text { hashtags })}+\frac{\sum_{d w=1}^{N_{d}} \log P\left(x_{d w}\right)}{\operatorname{count}(\operatorname{geotags})}\right)}$

The perplexity of the proposed LDA_Tags decreases when the number of topics has increased from 10 to 50. Comparison between LDA (only words distributions), LDA_hash (words and hashtags distributions), LDA_geo (words and geotags distributions) and LDA_Tags (words, hashtags and geotags distributions) with Chennai data shows that lower perplexity is achieved with LDA_Tags (Figure 7). However, it doesn't give the details about the statistical significance.

\section{Perplexity-Topic Models}

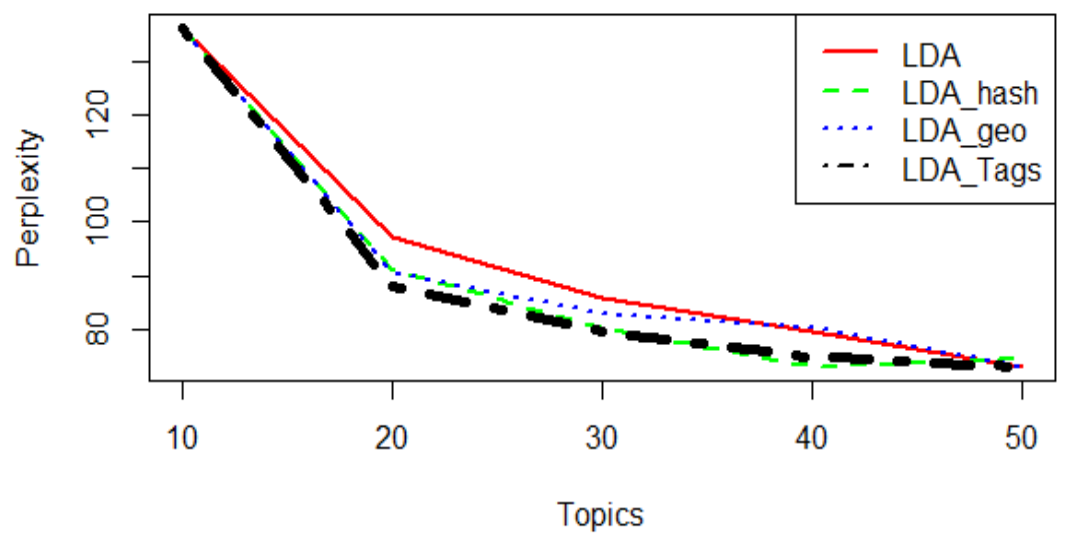

Figure 7. Perplexity vs. Topics

To find the significance of the topic model with tags context, one-way ANOVA analysis (Table 5) with Chennai data is done for the perplexity parameter over time. Since baseline LDA does 
not include tags, it is not considered for ANOVA analysis. The null hypothesis assumed here is "The mean of perplexities over three intervals is same for all topic models". The perplexity mean of three topic models (Table 5) over three periods are statistically different between groups with the minimum value of (143.2254) for LDA_Tags.

Table 5. One-way ANOVA for Topic Models

\begin{tabular}{llll}
\hline Groups & LDA_hash & LDA_geo & LDA_Tags \\
\hline $\mathrm{N}$ & 3 & 3 & 3 \\
\hline$\sum \mathrm{X}$ & 522.2208 & 515.083 & 429.6761 \\
\hline Mean & 174.0736 & 171.6943 & $\mathbf{1 4 3 . 2 2 5 4}$ \\
\hline$\sum \mathrm{X}^{2}$ & 90961.8053 & 88471.499 & 61563.9599 \\
\hline Std.Dev. & 5.3362 & 4.1633 & 3.4237 \\
\hline
\end{tabular}

It is also found that the F-measure between groups (Table 6) is 46.0966 and the p-value is less than 0.05 (0.0002). Hence, the hypothesis is rejected. P-values from within groups details, clearly show that LDA_Tags is significant compared to others and gives improved performance for tags based context.

Table 6. ANOVA - Group Details

\begin{tabular}{llllll}
\hline Source & Sum of Squares & Df & Variance & F measure & p-value \\
\hline Between-Groups & 1767.7508 & 2 & 883.8754 & 46.0966 & $\mathbf{0 . 0 0 0 2}$ \\
\hline Within-Groups & 115.0466 & 6 & 19.1744 & \\
\hline Total & 1882.7974 & 8 & & \\
\hline & & Within Group & Details & p-value \\
\hline Group & Group & Diff & Confidence & 0.7910 \\
\hline LDA_hash & LDA_geo & -2.3793 & $95 \%$ CI=-13.3494 to 8.5908 & $\mathbf{0 . 0 0 0 1}$ \\
\hline LDA_hash & LDA_Tags & -30.8482 & $95 \%$ CI=-41.8183 to -19.8781 & $\mathbf{0 . 0 0 0 3}$ \\
\hline LDA_geo & LDA_Tags & 28.4689 & $95 \% C I=-39.4390$ to -17.4988 & $\mathbf{0 . 0 1}$ \\
\hline
\end{tabular}

Log posterior estimates of the probability distributions of words, hashtags and geolocations vary with time (Figures 8a, 8b), which confirm their dynamic nature and the need for varying minibatch sizes. There is more variation in the distribution of words compared to hashtags and 
geolocations in Chennai data (Figure 8a). However, hashtags have more dynamic variation in distribution compared to the nominal change in words and geolocations in UK data (Figure 8b). This may be due to the abundant and frequent usage of social media by Twitter users in UK compared to Chennai.

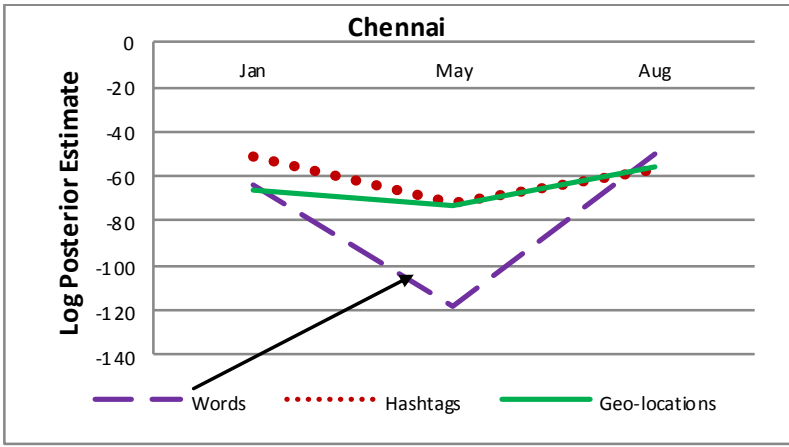

(a)

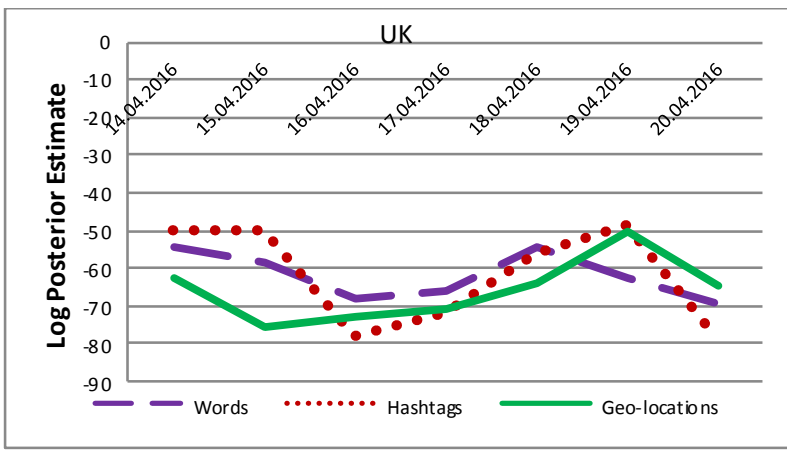

(b)

Figure 8. Posterior Estimate Variation

\subsubsection{Dynamic Model Evaluation}

The relative perplexity of recent with previous intervals (Knights et al. 2009) is calculated as,

Relative Perplexity $=1-\frac{\text { Perplexity }_{\text {recent }}}{\sum \text { Perplexity } \text { previous }}$

Performance of dynamic topic model with varying mini-batch sizes (words $-m_{w}^{t}$, hashtags $-m_{h}^{t}$, geolocations - $m_{g}^{t}$ ) is compared with that of random and fixed (words, hashtags, geolocations 100) mini-batch sizes. The average perplexity varies nearly at the same rate and lower values are obtained for SGLD_vm compared to that of SGLD_fm and SGLD_rm (Figure 9a). However, the relative perplexity is high for SGLD_vm with Chennai data and there is no distinction between the dynamic models with UK data which is due to the smaller interval between snapshots. Hence, SGLD_vm gives better performance compared to other models. 


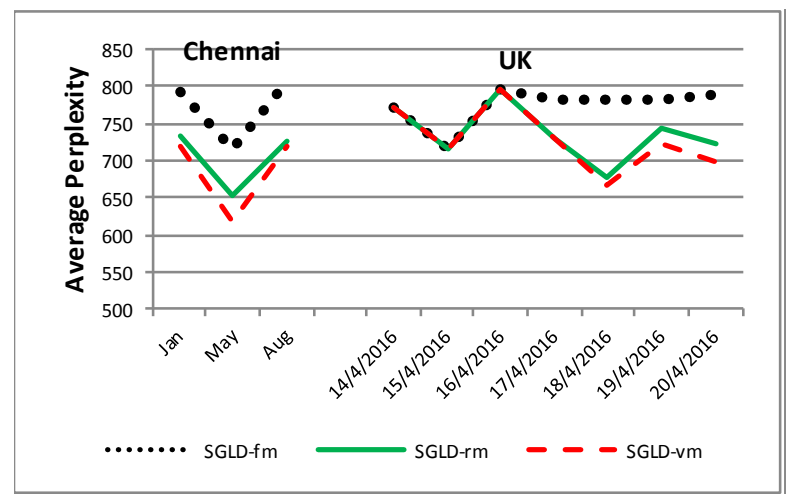

(a) Average Perplexity vs. Time

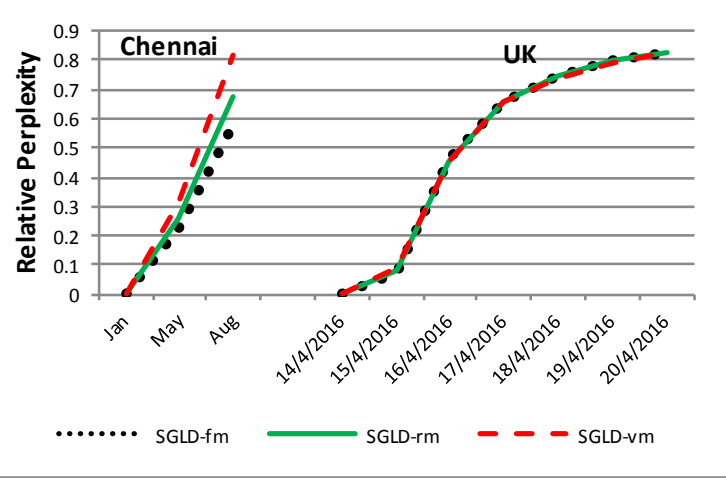

(b) Relative Perplexity vs. Time)

Figure 9. Dynamic Model Evluation

\subsubsection{Topical Word Embedding Evaluation}

Evaluation of TWE is achieved by visualizing the embedding vectors of top 5 topics using t-SNE distribution (Figure 10). The plot of TWEs without joint learning using topics from LDA (Figure 10a), LDA_hash (Figure 10b), LDA_geo (Figure 10c) and with joint learning (LDA_Tags) show that better grouping of topics is achieved with LDA_Tags (Figure 10d). 


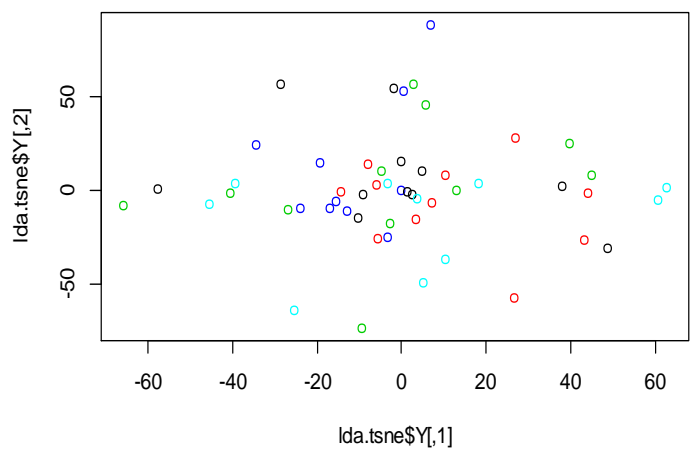

a. $L D A$

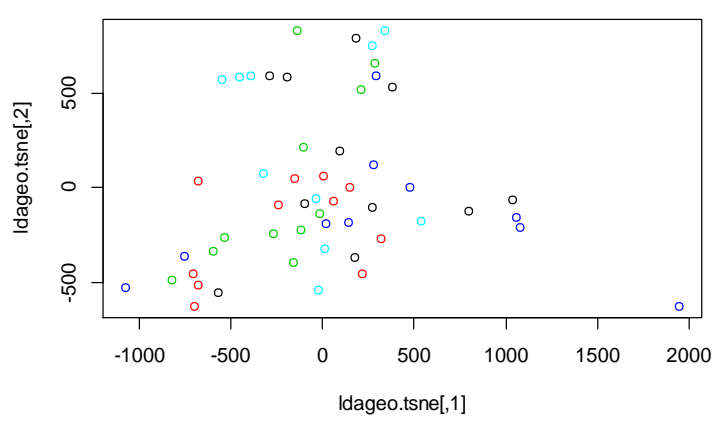

c. $L D A \_g e o$

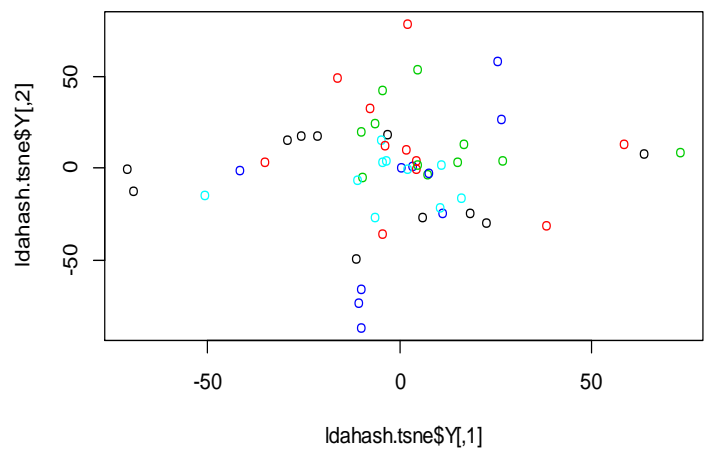

b. LDA_hash

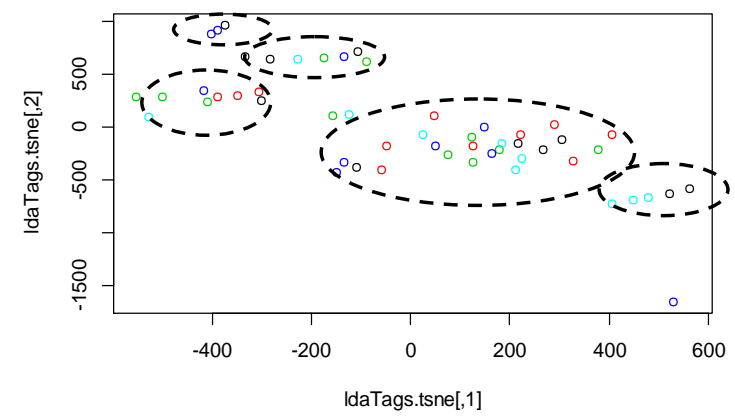

d. Joint Learning - LDA_Tags

\section{Figure 10. Topical Word Embedding Visualization - t_SNE}

\subsubsection{Topic Drift Evaluation}

Topic drift is evaluated by analyzing the KL divergence of topic's distribution (Equation 20) and MSD of topical embedding vectors over time (Equation 24). KL divergence among top ten topics of Chennai (college, jallikattu, actor, gonna, mersal, Ganesh Chaturthi, baahubali, beefban, chennaisilks, vivegam) and UK (Pressure, Humidity, Rain, Job, Wind, Weather, London, Books, Music and Sales) are plotted (Figures 11a \& 11b) for drift analysis. The divergence of topics 'jallikattu' and 'gonna' are high during January and August, and the topic 'actor' during May (Figure 11a). Among three topics, 'jallikattu' is rigorously affected by drift. Similarly the topic 'pressure' from UK data has moderate divergence on 20-4-16 and 'job' has 
very high divergence on 19-4-16 and 20-4-16 (Figure 11b) showing the impact of drift on 'job'. This is due to the association of 'job' with hashtag '\#o2job'.

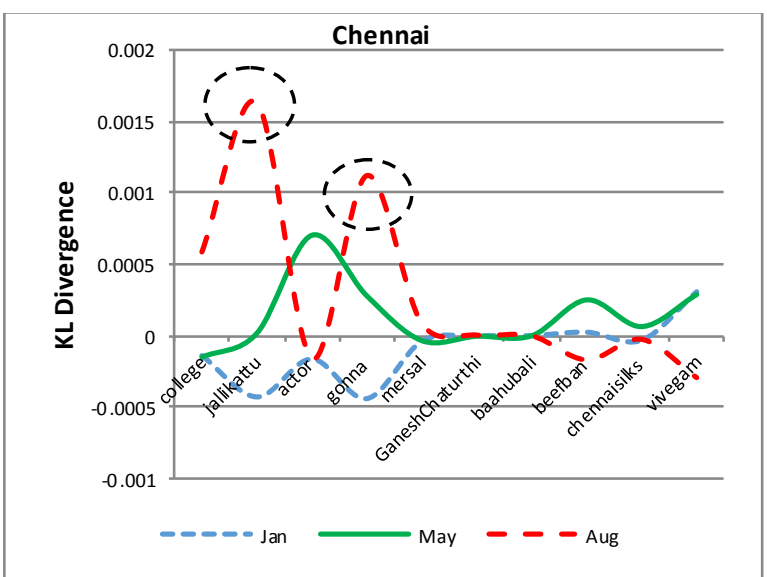

(a)

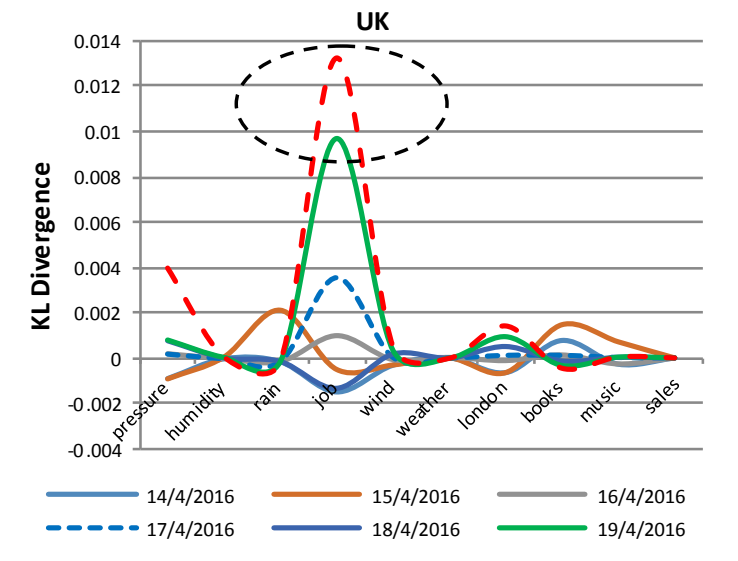

(b)

Figure 11. KL Divergence of Topics

The mean squared deviation of topical embedding vectors of ten topics over time shows that topics are having less deviation during May compared to January and August (Figure 12a). However, MSD of the topic 'jallikattu' is high during January (Figure 12a) which is the correct prediction of the duration of the protest compared to that of low divergence value (Figure 11a). KL divergence also wrongly detects drift in 'gonna' and 'actor' which is not actually true.

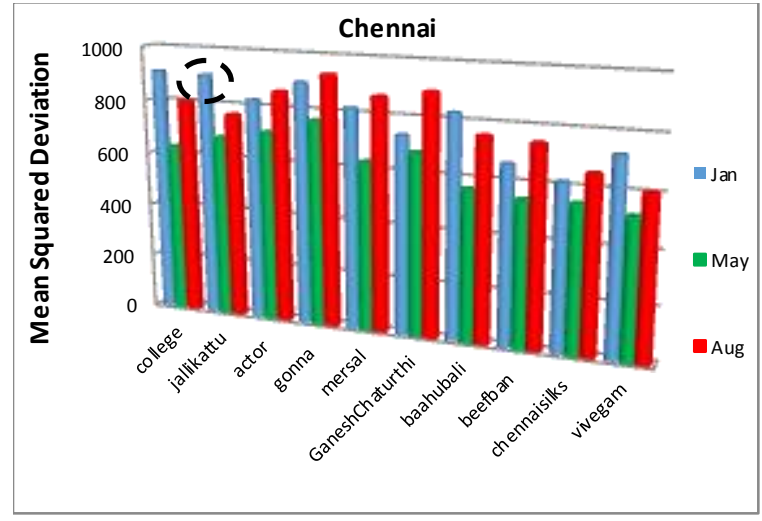

(a)

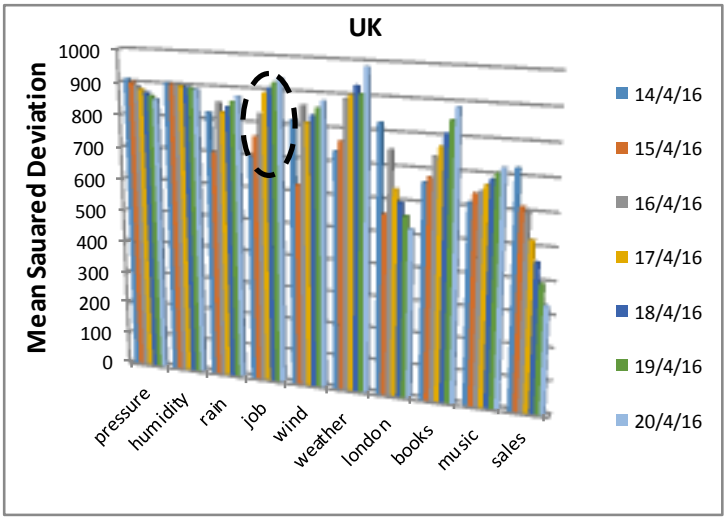

(b)

Figure 12. MSD Variation 
With UK data, the topics 'job', 'wind', 'weather' and 'books' have left side skew and 'london' and 'sales' have right side skew showing indications of the possibility of drift (Figure 12a). Among these 6 topics 'sales' has more variation denoting the drift in 'sales' during the interval. This is due to the co-occurrence of 'sales' with '\#internet'. The proposed topical embedding model correctly predicts drift in 'sales' whereas KL divergence shows no deviation from the topic 'sales' (Figure 12b). To confirm the results, TWEs of Chennai data (January) have been examined for topical variation of 'jallikattu'. Visualization using t-SNE (Figure 13) clearly shows the grouping of 'marina' with the topic 'jallikattu' due to the trends in the Twitter data during the protest.

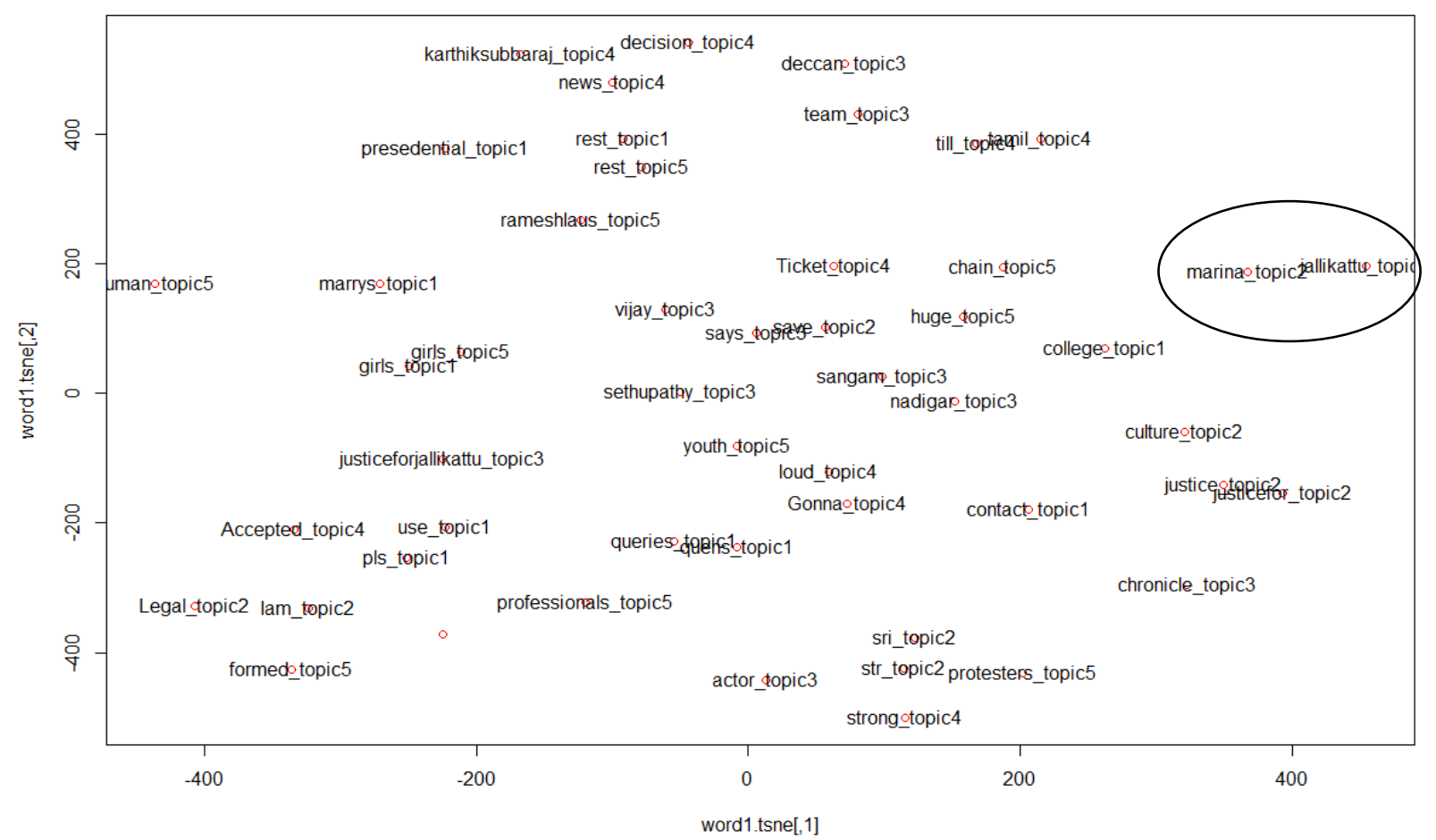

Figure 13. Topical Word Embedding - t-SNE

This ensures that the proposed model detects the drift in topics accurately compared to other models based on topic distribution over words and hashtags. This is possible with the joint learning of hashtags and geotags context. The topic drift detection accuracy of models based on contexts of only words (LDA), words+hashtags (LDA_hash), words+geotags (LDA_geo) and words+hashtags+geotags (LDA_Tags) have been compared for top 100 topics with different mini-batch size options (Figure 14). 


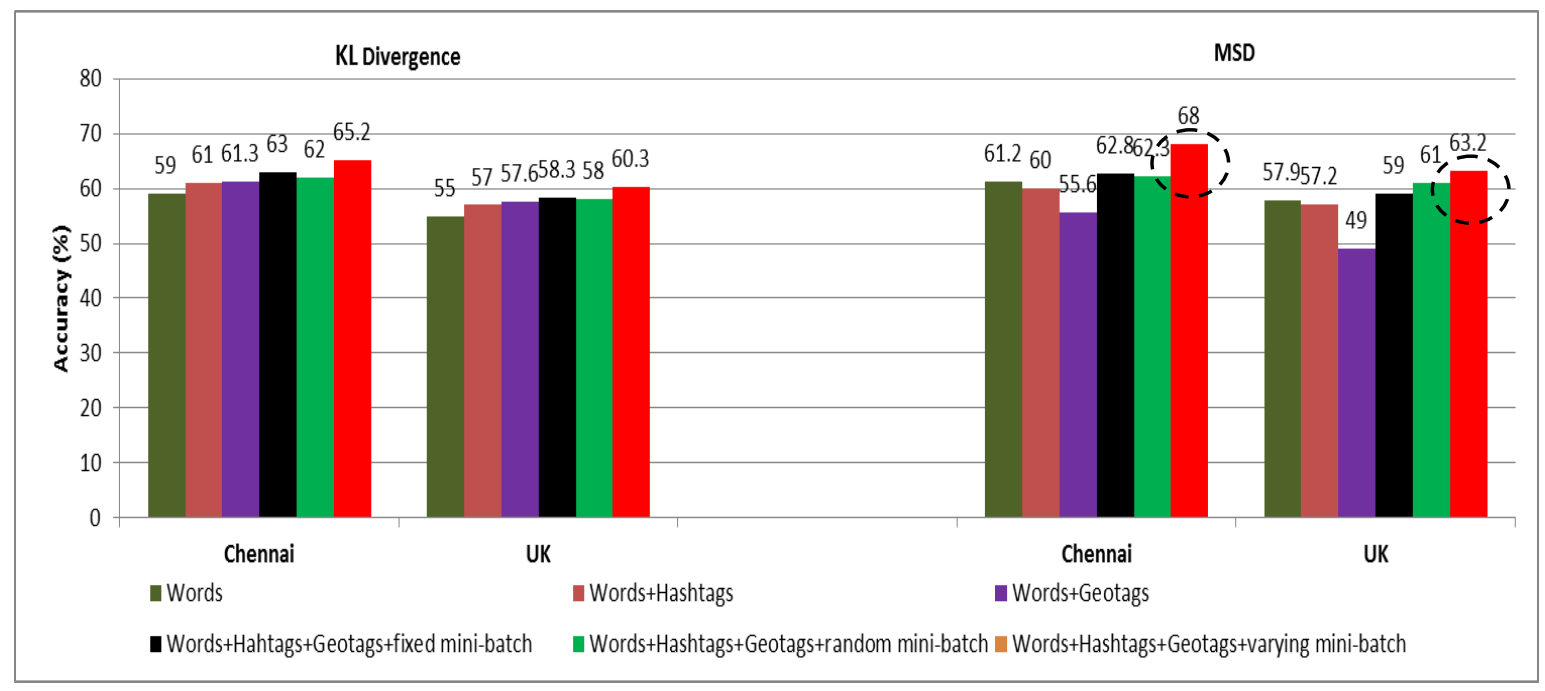

Figure 14. Accuracy

It is found that higher accuracy (68\% for Chennai data and $63.2 \%$ for UK data) is achieved with the proposed joint learning of TWE model with hashtags and geotags and with varying minibatch sizes compared with the other context models .

\section{CONCLUSION}

The proposed topical embedding model based on tags context represents topics and words in same semantic space effectively. The dynamic topic model SGLD with varying minibatch sizes performs well for computing the dynamic topic parameters with lower average perplexity and with higher relative perplexity for identifying the topic drift in the social media text with tags as topic indicators. Contextual changes in the topic distribution with hashtags and geolocations are better detected by the proposed topic model with tags contexts. MSD of topical embedding vectors detects the topic drift exactly than KL divergence of topic distribution. It can be extended in the future by adding different time level discretization for data ie. coarse level for words and geotags, and fine level for hashtags. Individual hashtags can be replaced by hashtag groups to accommodate more hashtags in the topical embedding model. Geolocations may also be grouped to study the topic change due to the community groups. Other cultural factors and events can also be related to the location based impact on the drift.

Conflict of interest: All authors declare that they have no conflict of interest. 


\section{REFERENCES}

1. Alam MH, Ryu WJ, Lee S. Context over time: Modeling context evolution in social media. In Proc 3rd Workshop on Data-Driven User Behavioral Modeling and Mining from Social Media. ACM. 2014;15-18.

2. Alam MH, Ryu WJ, Lee S. Hashtag-based topic evolution in social media. World Wide Web. 2017. 1-23.

3. Atefeh F, Khreich W. A Survey of Techniques for Event Detection in Twitter. Computational Intelligence. 2015. 132-164.

4. Bernabe-Moreno J, Tejeda-Lorente A, Porcel C, Herrera-Vied ma EA. New model to quantify the impact of a topic in a location over time with Social Media. Expert Systems with Applications. 2015; 42(7), 3381-3395.

5. Bhadury A, Chen J, Zhu J, Liu S. Scaling up dynamic topic models. In Proc of the 25th Int Conf on World Wide Web, International World Wide Web Conferences Steering Committee. 2016. 381-390.

6. Bian J, Gao B, Liu TY. Knowledge-powered deep learning for word embedding. In Joint European Conf on Machine Learning and Knowledge Discovery in Databases. Springer, Berlin, Heidelberg. 2014;132-148.

7. Blei DM, Ng AY, Jordan MI. Latent dirichlet allocation. Journal of machine Learning research. 2003;993-1022.

8. Blei DM, Lafferty JD. Dynamic topic models. In Proc of the 23rd int conf on Machine learning ACM. 2006;113-120.

9. Cai G, Peng L, Wang Y. Topic detection and evolution analysis on microblog. In Int Conf on Intelligent Information Processing. Springer, Berlin, Heidelberg. 2014 October; 67-77.

10. Cataldi M, Di Caro L, Schifanella C. Emerging topic detection on twitter based on temporal and social terms evaluation. In Proc of the tenth int workshop on multimedia data mining. ACM. 2010. 
11. Fei Y, Hong Y, Yang J. Handling topic drift for topic tracking in microblogs. In European Conf on Information Retrieval. Springer, Cham. 2015;477-488.

12. Griffiths TL, Steyvers M, Tenenbaum JB. Topics in semantic representation. Psychological review. 114(2), 2007;211.

13. Iwata T, Yamada T, Sakurai Y, Ueda N. Online multiscale dynamic topic models. In Proc of the 16th ACM SIGKDD int conf on Knowledge discovery and data mining. ACM. 2010;663-672.

14. Jiang D, Shi L, Lian R, Wu H. Latent Topic Embedding. In COLING. 2016;2689-2698.

15. Knights D, Mozer MC, Nicolov N. Detecting Topic Drift with Compound Topic Models. In ICWSM. 2009 March.

16. Li S, Huang G, Tan R, Pan R. Tag-weighted dirichlet allocation. In Data Mining (ICDM), 2013 IEEE 13th Int Conf on Data Mining. 2013;438-447.

17. Li M, Zhang T, Chen Y, Smola AJ. Efficient mini-batch training for stochastic optimization. In Proc of the 20th ACM SIGKDD int conf on Knowledge discovery and data mining. ACM. 2014;661-670.

18. Li X, Chi, J, Li C, Ouyang J, Fu B. Integrating Topic Modeling with Word Embeddings by Mixtures of vMFs. In COLING. 2016;151-160.

19. Liu Q, Huang H, Feng C. Micro-blog post topic drift detection based on LDA model. In Behavior and Social Computing. Springer, Cham. 2013;106-118.

20. Liu Y, Liu Z, Chua TS, Sun M. Topical Word Embeddings. In AAAI. 2015; 2418-2424.

21. Lozano MG, Schreiber J, Brynielsson J. Tracking geographical locations using a geo-aware topic model for analyzing social media data. Decision Support Systems. 99, 2017;18-29.

22. Mehrotra R, Sanner S, Buntine W, Xie L. Improving lda topic models for microblogs via tweet pooling and automatic labeling. In Proc of the 36th int ACM SIGIR conf on Research and development in information retrieval. ACM. 2013;889-892. 
23. Morchid, M, Josselin D, Portilla Y, Dufour R, Altman E, Linarès GA. Topic Modeling Based Representation to detect Tweet Locations. Example of the Event "JeSuis Charlie". Paper presented at the , Xl(3) 2015;629-634. doi:http://dx.doi.org/10.5194/isprsarchives-XL-3W3-629.

24. Niu L, Dai X, Zhang J, Chen J. Topic2Vec: learning distributed representations of topics. In Asian Language Processing (IALP), 2015 Int Conf on Asian Language Processing. IEEE. 2015;193-196.

25. Niu L, Dai XY, Huang S, Chen J. A unified framework for jointly learning distributed representations of word and attributes. In Asian Conf on Machine Learning. 2016;143156.

26. Qiang J, Chen P, Wang T, Wu X. Topic Modeling over Short Texts by Incorporating Word Embeddings. In Pacific-Asia Conf on Knowledge Discovery and Data Mining. Springer, Cham. 2017;363-374.

27. Quercia D, Askham H, Crowcroft J. TweetLDA: supervised topic classification and link prediction in Twitter. In Proceedings of the 4th Annual ACM Web Science Conference. 2012;247-250.

28. Ramage D, Hall D, Nallapati, R, Manning, CD. Labeled LDA: A supervised topic model for credit attribution in multi-labeled corpora. In Proc of the 2009 Conf on Empirical Methods in Natural Language Processing. Association for Computational Linguistics. Volume 1-Volume 1 2009;248-256.

29. Ramage D, Dumais ST, Liebling DJ. Characterizing microblogs with topic models. ICWSM. 10, 2010;1-1.

30. Rosa KD, Shah R, Lin B, Gershman A, Frederking R. Topical clustering of tweets. Proc of the ACM SIGIR: SWSM. 2011.

31. Rosen-Zvi M, Griffiths T, Steyvers M, Smyth P. The author-topic model for authors and documents. In Proc of the 20th conf on Uncertainty in artificial intelligence. AUAI Press. 2004;487-494. 
32. Saha A, Sindhwani V. Learning evolving and emerging topics in social media: a dynamic nmf approach with temporal regularization. In Proc of the fifth ACM int conf on Web search and data mining. ACM. 2012;693-702.

33. Tang J, Zhang M, Mei Q. One theme in all views: modeling consensus topics in multiple contexts. In Proc of the 19th ACM SIGKDD int conf on Knowledge discovery and data mining. 2013;5-13.

34. Wang C, Blei D, Heckerman D. Continuous time dynamic topic models. arXiv preprint arXiv. 2006;1206.3298.

35. Wang X, McCallum A. Topics over time: a non-Markov continuous-time model of topical trends. In Proc of the 12th ACM SIGKDD int conf on Knowledge discovery and data mining. 2006;424-433.

36. Wang Y, Liu J, Qu J, Huang Y, Chen J, Feng X. Hashtag graph based topic model for tweet mining. In Data Mining (ICDM), 2014 IEEE Int Conf on Data Mining. IEEE. 2014;10251030.

37. Wang Y, Liu J, Huang Y, Feng X. Using hashtag graph-based topic model to connect semantically-related words without co-occurrence in microblogs. IEEE Trans on Knowledge and Data Engineering. 2016;28(7), 1919-1933.

38. Welling M, Teh YW. Bayesian learning via stochastic gradient Langevin dynamics. In Proc of the 28th Int Conf on Machine Learning (ICML-11). 2011;681-688.

39. Xun G, Gopalakrishnan V, Ma F, Li Y, Gao J, Zhang A. Topic Discovery for Short Texts Using Word Embeddings. In Data Mining (ICDM), 2016 IEEE 16th Int Conf on Data Mining. IEEE. 2016;1299-1304.

40. Yang JG, Donnat C. CS 224 N: Language Dynamics analysis through Word2Vec Embeddings. 2017.

41. Zhang L, Sun X, Zhuge H. Topic discovery of clusters from documents with geographical location. Concurrency and Computation: Practice and Experience, 2015;27(15), 40154038. 
42. Zhao WX, Jiang J, Weng $\mathrm{J}$ et al. Comparing twitter and traditional media using topic models. In European Conf on Information Retrieval. Springer, Berlin, Heidelberg. 2011;338-349. 


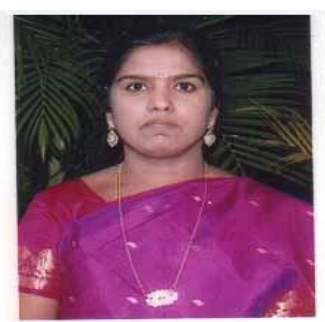

J Vijayarani is currently doing Ph.D at Anna University Chennai. Her research interests include NLP, Text Mining, Semantic Computing and Deep Learning. Address for correspondence: Teaching Fellow, CSE Department, CEG Campus, Anna University, Chennai. Email:viji.cs66@gmail.com

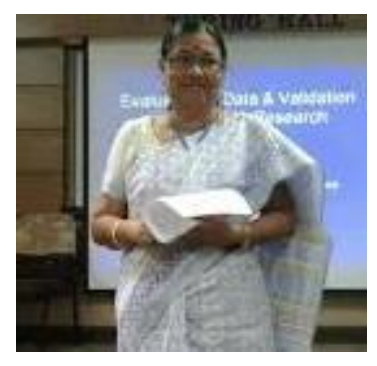

Dr T V Geetha completed her Ph.D in Natural Language Processing from Anna University and is currently Retd Senior Professor and UGC-BSR Faculty Fellow, Computer Science and Engineering Department, CEG Campus, Anna University, Chennai. Her research interests include Biomedical Text Mining, Music Processing, Tamil Computing, Text Mining, Search Engines, Semantic Computing, Deep Learning etc. Email: tv_g@hotmail.com 